\title{
Searching for QCD instantons at hadron colliders
}

\author{
Valentin V. Khoze $\odot,^{*}$ Daniel L. Milne, ${ }^{\dagger}$ and Michael Spannowsky \\ IPPP, Department of Physics, Durham University, Durham DH1 3LE, United Kingdom
}

(Received 12 October 2020; accepted 1 December 2020; published 19 January 2021)

\begin{abstract}
QCD instantons are arguably the best motivated yet unobserved nonperturbative effects predicted by the Standard Model. A discovery and detailed study of instanton-generated processes at colliders would provide a new window into the phenomenological exploration of QCD and a vastly improved fundamental understanding of its nonperturbative dynamics. Building on the optical theorem, we numerically calculate the total instanton cross section from the elastic scattering amplitude, also including quantum effects arising from resummed perturbative exchanges between hard gluons in the initial state, thereby improving in accuracy on previous results. Although QCD instanton processes are predicted to be produced with a large scattering cross section at small center-of-mass partonic energies, discovering them at hadron colliders is a challenging task that requires dedicated search strategies. We evaluate the sensitivity of high-luminosity LHC runs, as well as low-luminosity LHC and Tevatron runs. We find that LHC low-luminosity runs in particular, which do not suffer from large pileup and trigger thresholds, show a very good sensitivity for discovering QCD instanton-generated processes.
\end{abstract}

DOI: 10.1103/PhysRevD.103.014017

\section{INTRODUCTION}

Instantons are arguably the best motivated nonperturbative effects in the Standard Model (SM), and yet they have not been observed so far. Our motivation in this paper is to reexamine QCD instanton contributions to high-energy scattering processes at hadron colliders building up on the recent work [1] in establishing a robust QCD instanton computational formalism focused on applications to proton colliders and to discuss experimental signatures.

The status of the SM as the theory of the currently accessible fundamental interactions in particle physics is well established. To a large extent, the evidence for the SM as the most precise theoretical framework for describing strong and electroweak interactions comes from comparing perturbative calculations with the data from particle experiments. The reliance on the weakly coupled perturbation theory is justified at high energies thanks to the asymptotic freedom in the Yang-Mills theory. But there is another consequence of the non-Abelian nature of the theory that necessitates an inclusion of nonperturbative effects. The non-Abelian nature of QCD and of the weak interactions is

\footnotetext{
*valya.khoze@durham.ac.uk

daniel.1.milne@durham.ac.uk

michael.spannowsky@durham.ac.uk
}

Published by the American Physical Society under the terms of the Creative Commons Attribution 4.0 International license. Further distribution of this work must maintain attribution to the author(s) and the published article's title, journal citation, and DOI. Funded by SCOAP ${ }^{3}$. known to give rise to a rich vacuum structure in the Standard Model. This vacuum structure is well understood in the semiclassical picture $[2,3]$ and amounts to augmenting the perturbative vacuum with an infinite set of topologically nontrivial vacuum sectors in a Yang-Mills theory.

Instanton field configurations [4] are classical solutions of Yang-Mills equations of motion in the Euclidean space which interpolate between the different semiclassical vacuum sectors in the theory. At weak coupling instantons provide dominant contributions to the path integral and correspond to quantum tunneling between different vacuum sectors of the SM. These effects are beyond the reach of ordinary perturbation theory, and in particular in the electroweak theory they lead to the violation of baryon plus lepton number $(\mathrm{B}+\mathrm{L})$, while in $\mathrm{QCD}$ instantons processes violate chirality $[5,6]$,

$$
g+g \rightarrow n_{g} \times g+\sum_{f=1}^{N_{f}}\left(q_{R f}+\bar{q}_{L f}\right),
$$

where $N_{f}$ is the number of light (i.e., nearly massless relative to the energy scale probed by the instanton) quark flavors. The QCD instanton-generated process (1.1) with two gluons in the initial state going to an arbitrary number of gluons in the final state along with $2 N_{f}$ quarks will be the focus of our discussion in Sec. II.

The purpose of this paper is to provide the most up-todate computationally robust calculation of QCD instanton contributions to high-energy scattering processes relevant 
for hadron colliders. At the level of the partonic instanton cross section, there are two main ingredients in the approach we follow. We shall use the optical theorem approach that will effectively allow us to sum over all final states with an arbitrary number of gluons. This is achieved by evaluating the imaginary part of the forward elastic scattering amplitude computed in the background of the instanton-anti-instanton configuration. This formalism was originally developed in [7] based on the instanton-antiinstanton field configuration constructed in [8].

The second ingredient of our approach relies on the inclusion of certain higher-order effects in the instanton perturbation theory. Specifically we will take into account resummed radiative exchanges between the hard partons in the initial state $[9,10]$, as they provide the dominant contribution to breaking the classical scale invariance of QCD in quantum theory. Inclusion of these quantum effects (often referred to in the instanton literature as the hard-hard quantum corrections) is required in order to resolve the well-known nonperturbative infrared (IR) problem that arises from contributions of QCD instantons with large scale sizes, as was first shown in [1]. We will see that contributions of QCD instantons with large sizes are automatically cut off by the inclusion of these quantum effects.

To a large extent the theory formalism we employ in this paper for computing QCD instanton rates is the same as in the earlier work [1], but we are able to carry out a more complete evaluation of instanton integrals without relying on the saddle-point approximation. Specifically, in Sec. II D we will numerically compute integrals over all instanton-anti-instanton collective coordinates that correspond to positive modes of the instanton-anti-instanton action. Only the final integration over the single negative mode that gives rise to the imaginary part of the amplitude, as required by the optical theorem, will be carried out in the saddle-point approximation. This provides a more robust prediction leading on average to an order of magnitude increase in instanton partonic cross sections in our case. There is also a number of other more minor technical improvements, in particular in relation to the computation of the mean number of gluons in the final state in Sec. II E. Our results summarized in Tables I and II present cross sections for instanton-generated processes at partonic and hadronic levels for the LHC and the Tevatron as well as for $30 \mathrm{TeV}$ and $100 \mathrm{TeV}$ future hadron colliders.

In Sec. III we explain how to generalize the calculation of the instanton process to the case where a jet is emitted from one of the initial state partons. We find that cross sections calculated for the processes where the instanton recoils against a jet with large momentum are too small to be observable at any present or envisioned high-energy collider. In order to obtain sensitivity to instantons is to disentangle their spherical radiation profile, made of fairly soft jets from the perturbative backgrounds.
The event topology of instanton events with its spherical energy distribution between a large number of final-state objects is visibly distinguishable from the usual few-jets events generated in perturbative-QCD processes at the LHC, as discussed in Sec. IVA, but QCD instanton processes occur predominantly at small partonic centerof-mass energies. The combination of both these characteristics suggests that QCD-instanton events are soft bombs [1], using the terminology of Ref. [11], where the phenomenology of such events was first investigated in the context of beyond the Standard Model physics. In our case the soft bombs are fully Standard Model made. At highenergy colliders, such events struggle to pass trigger and event reconstruction cuts. In Secs. IV B 1 and IV C we assess whether the comparably large hadronic instanton cross sections might give rise to visible signatures at hadron colliders, in particular the LHC or the Tevatron. Examination of data collected with a minimum bias trigger shows that it should be possible to either discover instantons or severely constrain their cross section. We conclude with a summary in Sec. V.

\section{COMPUTATION OF THE INSTANTON PARTONIC CROSS SECTION}

Instanton gauge fields $A_{\mu}^{\text {inst }}(x)$ are the solutions to the self-duality equation, $F_{\mu \nu}=\tilde{F}_{\mu \nu}$, and as such, instantons are local minima of the Euclidean action. In QCD, the instanton configuration contains the gauge field and the fermion components,

$A_{\mu}=A_{\mu}^{\mathrm{inst}}(x), \quad \bar{q}_{L f}=\psi^{(0)}(x), \quad q_{R f}=\psi^{(0)}(x)$,

where the gauge field $A_{\mu}^{\text {inst }}$ is the instanton solution [4] of topological charge 1 ,

$$
A_{\mu}^{a \text { inst }}(x)=\frac{2 \rho^{2}}{g} \frac{\bar{\eta}_{\mu \nu}^{a}\left(x-x_{0}\right)_{\nu}}{\left(x-x_{0}\right)^{2}\left(\left(x-x_{0}\right)^{2}+\rho^{2}\right)},
$$

and the constants $\bar{\eta}_{\mu \nu}^{a}$ are the 't Hooft eta symbols [5]. The fermionic components $\psi^{(0)}$ in (2.1) are known as the instanton fermion zero modes. They are given by the (nonvanishing) solutions of the Dirac equation in the $A_{\mu}^{a \text { inst }}$ instanton background, $\gamma^{\mu} D_{\mu}^{\text {inst }} \psi^{(0)}=0$. The Euclidean action of the BPST instanton is

$$
S\left[A_{\mu}^{\mathrm{inst}}\right]=S_{I}=\frac{8 \pi^{2}}{g^{2}}=\frac{2 \pi}{\alpha_{s}\left(\mu_{r}\right)},
$$

where for the later convenience we have included the dependence of the coupling constant on the renormalisation group (RG) scale $\mu_{r}$. For more details on instantons and their applications relevant to the material in this section, an interested reader can consult a selection of review articles in Refs. [12-16]. Our presentation in Secs. II A and II B 
follows a recent overview of QCD instanton calculus in Ref. [1].

\section{A. QCD instantons and scattering amplitudes}

The scattering amplitude for the $2 \rightarrow n_{g}+2 N_{f}$ instanton-generated process (1.1) is computed by expanding the path integral around the instanton field configuration (2.1).

The amplitude takes the form of an integral over the instanton collective coordinates,

$$
\begin{aligned}
\mathcal{A}_{2 \rightarrow n_{g}+2 N_{f}}= & \int d^{4} x_{0} \int_{0}^{\infty} d \rho D(\rho) e^{-S_{I}} \prod_{i=1}^{n_{g}+2} A_{\mathrm{LSZ}}^{\mathrm{inst}}\left(p_{i} ; \rho\right) \\
& \times \prod_{j=1}^{2 N_{f}} \psi_{\mathrm{LSZ}}^{(0)}\left(p_{j} ; \rho\right) .
\end{aligned}
$$

The integral (2.4) is over the instanton position $x_{0}^{\mu}$ and the scale-size collective coordinate $\rho$, and it involves the instanton density function $D(\rho)$, the semiclassical suppression factor $e^{-S_{I}}$ by the instanton action (2.3), and the product of vector boson and fermion field configurations, one for each external leg of the amplitude, computed on the instanton solutions, and Lehmann-SymanzikZimmermann (LSZ) reduced.

The instanton density $D(\rho)$ in (2.4) arises from computing quadratic fluctuation determinants in the instanton background in the path integral. This is a one-loop effect in the perturbation theory around the instanton, and the result is given by [5]

$$
D\left(\rho, \mu_{r}\right)=\kappa \frac{1}{\rho^{5}}\left(\frac{2 \pi}{\alpha_{s}\left(\mu_{r}\right)}\right)^{2 N_{c}}\left(\rho \mu_{r}\right)^{b_{0}},
$$

where $\kappa$ is the normalization constant of the instanton density in the $\overline{\mathrm{MS}}$ scheme [17-19],

$$
\begin{aligned}
\kappa & =\frac{2 e^{5 / 6-1.511374 N_{c}}}{\pi^{2}\left(N_{c}-1\right) !\left(N_{c}-2\right) !} e^{0.291746 N_{f}} \\
& \simeq 0.0025 e^{0.291746 N_{f}}
\end{aligned}
$$

and $b_{0}=(11 / 3) N_{c}-(2 / 3) N_{f}$.

Expressions for the LHZ-reduced instanton field insertions on the right-hand side (RHS) of the integral in (2.4) are obtained from the momentum-space representation of the instanton solution (2.2),

$$
\begin{aligned}
A_{\mathrm{LSZ}}^{a \text { inst }}(p, \lambda) & =\lim _{p^{2} \rightarrow 0} p^{2} \epsilon^{\mu}(\lambda) A_{\mu}^{a \text { inst }}(p) \\
& =\epsilon^{\mu}(\lambda) \bar{\eta}_{\mu \nu}^{a} p_{\nu} \frac{4 i \pi^{2} \rho^{2}}{g} e^{i p \cdot x_{0}},
\end{aligned}
$$

where $\epsilon^{\mu}(\lambda)$ is the polarization vector for a gluon with a helicity $\lambda$. A similar expression also holds for the LSZ- amputated fermion zero modes, in this case, $\psi_{\mathrm{LSZ}}^{(0)} \propto \rho$ rather than $A_{L S Z}^{\text {inst }} \propto \rho^{2}$ for the gauge field.

Combining all the ingredients above, it is now easy to see that the $\rho$ integral in the leading-order instanton amplitude (2.4) is powerlike divergent—a well-known fact that signals the breakdown of the leading-order instanton calculation in QCD at large distances $(\rho \gtrsim 1 / \Lambda)$ where the coupling becomes strong and the semiclassical approximation is invalidated. Instantons are solutions to classical equations, and unless quantum effects due to field fluctuations around instantons are appropriately taken into account, there is no scale in the microscopic QCD Lagrangian to cut off large values of the instanton size $-\rho$ is a classically flat direction. To break classical scale invariance we need to include quantum corrections that describe interactions of the external states. This amounts to inserting propagators in the instanton background between pairs of external fields in the preexponential factor in (2.4) and resumming the resulting perturbation theory. The dominant effect comes from interactions between the two initial hard gluons [9] (these are the states that carry the largest kinematic invariant $p_{1} \cdot p_{2}=\hat{s} / 2$ ). In Ref. [10] Mueller has shown that these quantum corrections formally exponentiate and the resulting expression for the resummed quantum corrections around the instanton generates the factor

$$
e^{-\left(\alpha_{s}\left(\mu_{r}\right) / 16 \pi\right) \rho^{2} E^{2} \log E^{2} / \mu_{r}^{2}},
$$

where $E$ is the partonic centre of mass (CoM) energy, $E^{2} \equiv \hat{s}$. This exponential factor provides an automatic cutoff of the large instanton sizes, and the instanton integral over $\rho$ can now be safely evaluated.

To proceed, we need to select a value for the renormalization scale $\mu_{r}$. Recall that the integrand in (2.4) contains the factor

$$
\left(\rho \mu_{r}\right)^{b_{0}} e^{-\frac{2 \pi}{\alpha_{s}\left(\mu_{r}\right)}}=e^{-\frac{2 \pi}{\alpha_{s}(1 / \rho)}}
$$

where $\left(\rho \mu_{r}\right)^{b_{0}}$ comes from the instanton density and the factor $e^{-\frac{2 \pi}{\alpha_{S}\left(\mu_{r}\right)}}$ accounts for the contribution of the instanton action $S_{I}=\frac{2 \pi}{\alpha_{s}\left(\mu_{r}\right)}$. The RHS of (2.10) is RG invariant at one loop, it does not depend on the choice of $\mu_{r}$, and instead the scale of the running coupling constant is set at the inverse instanton size. To take advantage of this and to remove large powers of $\rho$ from the integrand, from now on and until the end of this section, we will set the RG scale value at the instanton size,

$$
\mu_{r}=1 / \rho
$$

The amplitude integrand including Mueller's exponentiated quantum effect is given by 


$$
\begin{aligned}
\mathcal{A}_{2 \rightarrow n_{g}+2 N_{f}}= & \kappa \int d^{4} x_{0} \int_{0}^{\infty} \frac{d \rho}{\rho^{5}}\left(\frac{2 \pi}{\alpha_{s}}\right)^{6} e^{-\frac{2 \pi}{\alpha_{S}(1 / \rho)}-\frac{\alpha_{S}(1 / \rho)}{16 \pi} \rho^{2} E^{2} \log E^{2} \rho^{2}} \\
& \times \prod_{i=1}^{n_{g}+2} A_{\mathrm{LSZ}}^{\mathrm{inst}}\left(p_{i} ; \rho\right) \prod_{j=1}^{2 N_{f}} \psi_{\mathrm{LSZ}}^{(0)}\left(p_{j} ; \rho\right) .
\end{aligned}
$$

Keeping a careful track of the powers of $\rho$, the resulting integral in (2.11) is proportional to the following expression (we note that the integral over the instanton position $\int d^{4} x_{0}$ gives the delta function of the momentum conservation that we drop, along with the overall constant and $\rho$-independent factors):

$$
\mathcal{A}_{2 \rightarrow n_{g}+2 N_{f}} \sim \int_{0}^{\infty} d \rho\left(\rho^{2}\right)^{n_{g}+2+N_{f}-5 / 2} e^{-\frac{\alpha_{s}(1 / \rho)}{16 \pi} E^{2} \rho^{2} \log \left(E^{2} \rho^{2}\right)-\frac{2 \pi}{\alpha_{s}(1 / \rho)}} .
$$

The integral is no longer divergent in the IR limit of large $\rho$ and can be evaluated and the resulting expression for the amplitude can be used to compute the instanton cross section. In the following section we will obtain the instanton cross section in a more efficient manner using the optical theorem approach in the following section (Sec. II B).

Before we conclude this section, we would like to comment on the structure of the leading-order instanton expression (2.11). Note that the integrand on the right-hand side of (2.11) contains a simple product of bosonic and fermionic components of instanton field configurations, one for each external line of the amplitude. Such a fully factorized structure of the field insertions implies that at the leading order in instanton perturbation theory there are no correlations between the momenta of the external legs in the instanton amplitude. Emissions of individual particles in the final state are mutually independent, apart from the overall momentum conservation. The expression in (2.11) looks like a multiparticle pointlike vertex integrated over the instanton position and size. Thanks to its pointlike structure, the instanton vertex in the center-of-mass frame describes the scattering process into a spherically symmetric multiparticle final state. The number of gluons $n_{g}$ is unconstrained and can be as large as is energetically viable [20,21] (in practice, the dominant contribution will come from $\left\langle n_{g}\right\rangle \sim 4 \pi / \alpha_{s} \gg 1$ ), and a fixed number of quarks (a $q_{L} \bar{q}_{R}$ pair for each light quark flavor).

\section{B. The optical theorem approach}

To compute a total parton-level instanton cross section $\hat{\sigma}_{\text {tot }}^{\text {inst }}$ for the process $g g \rightarrow X$, we use the optical theorem to relate the cross section to the imaginary part of the forward elastic scattering amplitude computed in the background of the instanton-anti-instanton $(I \bar{I})$ configuration,

$$
\hat{\sigma}_{\mathrm{tot}}^{\mathrm{inst}}=\frac{1}{E^{2}} \operatorname{Im} \mathcal{A}_{4}^{I \bar{I}}\left(p_{1}, p_{2},-p_{1},-p_{2}\right),
$$

where $E=\sqrt{\hat{s}}=\sqrt{\left(p_{1}+p_{2}\right)^{2}}$ is the partonic CoM energy.

For the reader's convenience in Appendix A we outline main steps of the formalism to represent the forward elastic scattering amplitude as the integral over collective coordinates of the instanton-anti-instanton field configuration following the valley method approach developed in $[7,8,22-25]$.

For our purposes it is sufficient to simply note that the instanton-anti-instanton gauge field is a trajectory in the topological charge zero sector of the field configuration space parametrized by instanton and anti-instanton collective coordinates. This trajectory interpolates between the sum of infinitely separated instanton and anti-instanton and the perturbative vacuum,

$$
\begin{gathered}
R \rightarrow \infty: A_{\mu}^{I \bar{I}}(x) \rightarrow A_{\mu}^{I}\left(x-x_{0}\right)+A_{\mu}^{\bar{I}}\left(x-x_{0}-R\right), \\
R \rightarrow 0: A_{\mu}^{I \bar{I}}(x) \rightarrow 0 .
\end{gathered}
$$

The configuration $A_{\mu}^{I \bar{I}}(x)$ for arbitrary values of the collective coordinates is determined by solving the gradient flow equation known as the valley equation.

The collective-coordinate integral for the amplitude reads

$$
\begin{aligned}
\mathcal{A}_{4}^{I \bar{I}}\left(p_{1}, p_{2},-p_{1},-p_{2}\right) \\
=\int_{0}^{\infty} d \rho \int_{0}^{\infty} d \bar{\rho} \int d^{4} R \\
\quad \times \int d \Omega D(\rho) D(\bar{\rho}) e^{-S_{I \bar{I}}-\frac{\alpha_{s}}{16 \pi}\left(\rho^{2}+\bar{\rho}^{2}\right) E^{2} \log \frac{E^{2}}{\mu_{r}^{2}}} \\
\quad \times A_{\mathrm{LSZ}}^{\text {inst }}\left(p_{1}\right) A_{\mathrm{LSZ}}^{\mathrm{inst}}\left(p_{2}\right) A_{\mathrm{LSZ}}^{\overline{\text { inst }}}\left(-p_{1}\right) A_{\mathrm{LSZ}}^{\overline{\mathrm{inst}}}\left(-p_{2}\right) \mathcal{K}_{\text {ferm }} .
\end{aligned}
$$

In the expression above we integrate over all collective coordinates: $\rho$ and $\bar{\rho}$ are the instanton and anti-instanton sizes, $R_{\mu}=\left(R_{0}, \vec{R}\right)$ is the separation between the $I$ and $\bar{I}$ positions in the Euclidean space, and $\Omega$ is the $3 \times 3$ matrix of relative $I \bar{I}$ orientations in the $S U(3)$ colour space. $D(\rho)$ and $D(\bar{\rho})$ represent the instanton and the anti-instanton densities (2.5) and the field insertions $A_{\mathrm{LSZ}}^{\text {inst }}(p)$ and $A_{\mathrm{LSZ}}^{\overline{\mathrm{inst}}}\left(p^{\prime}\right)$ are the LSZ-reduced instanton and anti-instanton fields (2.7). For each pair of the gluon legs with the same incoming/outgoing momentum we have

$$
\begin{aligned}
& \frac{1}{3} \sum_{a=1}^{3} \frac{1}{2} \sum_{\lambda=1,2} A_{\mathrm{LSZ}}^{a \text { inst }}(p, \lambda) A_{\mathrm{LSZ}}^{a \overline{\text { inst }}}(-p ; \lambda) \\
& \quad=\frac{1}{6}\left(\frac{2 \pi^{2}}{g} \rho \bar{\rho} \sqrt{s^{\prime}}\right)^{2} e^{i R \cdot p},
\end{aligned}
$$

and now for the combination of all four external gluon insertions in (2.16) we have 


$$
\begin{aligned}
& A_{\mathrm{LSZ}}^{\mathrm{inst}}\left(p_{1}\right) A_{\mathrm{LSZ}}^{\mathrm{inst}}\left(p_{2}\right) A_{\mathrm{LSZ}}^{\overline{\text { inst }}}\left(-p_{1}\right) A_{\mathrm{LSZ}}^{\overline{\overline{\mathrm{nst}}}}\left(-p_{2}\right) \\
& \quad=\frac{1}{36}\left(\frac{2 \pi^{2}}{g} \rho \bar{\rho} \sqrt{s^{\prime}}\right)^{4} e^{i R \cdot\left(p_{1}+p_{2}\right)}
\end{aligned}
$$

The contribution $e^{i R \cdot\left(p_{1}+p_{2}\right)}$ arises from the exponential factors $e^{i p_{i} \cdot x_{0}}$ and $e^{-i p_{i} \cdot \bar{x}_{0}}$ from the two instanton and two anti-instanton legs, which upon the Wick rotation to the Minkowski space becomes $e^{R_{0} \sqrt{s^{\prime}}}$.

The final factor on the right-hand side of (2.16) (apart from the expression in the exponent) is the overlap of fermion zero modes $\mathcal{K}_{\text {ferm }}$, which we will define near the end of the section.

We now turn to the exponent in (2.16). The action of the instanton-anti-instanton configuration was computed in $[7,23,24]$, it is a function of a single variable $z$ known as the conformal ratio of the (anti-)instanton collective coordinates,

$z=\frac{R^{2}+\rho^{2}+\bar{\rho}^{2}+\sqrt{\left(R^{2}+\rho^{2}+\bar{\rho}^{2}\right)^{2}-4 \rho^{2} \bar{\rho}^{2}}}{2 \rho \bar{\rho}}$,

and it takes the form $S_{I \bar{I}}(z)=\frac{4 \pi}{\alpha_{s}} \mathcal{S}(z)$ where

$$
\begin{aligned}
\mathcal{S}(z)= & 3 \frac{6 z^{2}-14}{(z-1 / z)^{2}}-17 \\
& -3 \log (z)\left(\frac{(z-5 / z)(z+1 / z)^{2}}{(z-1 / z)^{3}}-1\right) .
\end{aligned}
$$

For more detail on the derivation of the instanton-antiinstanton valley trajectory and the plot of the action as the function of the interinstanton separation we refer the reader to Appendix A and Refs. [7,8,23,24].

The second term in the exponent in (2.16) is recognized as Mueller's quantum effect of the hard-hard gluon exchanges in the initial state (2.8) and the similar factor for the anti-instanton gluon exchanges in the final state.

The final factor appearing in (2.16) that needs to be defined is $\mathcal{K}_{\text {ferm }}(z)$. This simply comes from calculating the overlap between the instanton and anti-instanton fermion zero modes [26],

$$
\omega=\int d^{4} x \psi_{0}^{\bar{I}}(x) i \not D \psi_{0}^{I}(x) .
$$

The authors of Ref. [26] also found an integral expression for this which was then calculated analytically in [27], and this expression is then raised to the power $2 N_{f}$, the number of fermions. It arises from the $2 N_{f}$ fermions in the final state of the process (1.1). As the instanton-anti-instanton action function $\mathcal{S}(z)$, the fermion factor $\mathcal{K}_{\text {ferm }}(z)$ is a function of a single variable - the conformal ratio $z$ defined in (2.19). We have

$$
\mathcal{K}_{\text {ferm }}=\left(\omega_{\text {ferm }}\right)^{2 N_{f}},
$$

where $\omega_{\text {ferm }}(z)$ was computed in [27],

$$
\omega_{\text {ferm }}(z)=\frac{3 \pi}{8} \frac{1}{z^{3 / 2}}{ }_{2} F_{1}\left(\frac{3}{2}, \frac{3}{2} ; 4 ; 1-\frac{1}{z^{2}}\right) .
$$

Putting everything together we can now write down the instanton cross section (2.13) as the finite-dimensional integral in the form

$$
\begin{aligned}
\hat{\sigma}_{\mathrm{tot}}^{\text {inst }} \simeq & \frac{1}{E^{2}} \operatorname{Im} \frac{\kappa^{2} \pi^{4}}{36 \times 4} \int \frac{d \rho}{\rho^{5}} \int \frac{d \bar{\rho}}{\bar{\rho}^{5}} \int d^{4} R \int d \Omega\left(\frac{2 \pi}{\alpha_{s}\left(\mu_{r}\right)}\right)^{14}\left(\rho^{2} E\right)^{2}\left(\bar{\rho}^{2} E\right)^{2} \mathcal{K}_{\text {ferm }}(z) \\
& \times\left(\rho \mu_{r}\right)^{b_{0}}\left(\bar{\rho} \mu_{r}\right)^{b_{0}} \exp \left(R_{0} E-\frac{4 \pi}{\alpha_{s}\left(\mu_{r}\right)} \mathcal{S}(z)-\frac{\alpha_{s}\left(\mu_{r}\right)}{16 \pi}\left(\rho^{2}+\bar{\rho}^{2}\right) E^{2} \log \frac{E^{2}}{\mu_{r}^{2}}\right) .
\end{aligned}
$$

To further simplify the integrand we would like to select a natural value for the renormalization scale that removes the $\left(\rho \mu_{r}\right)^{b_{0}}\left(\bar{\rho} \mu_{r}\right)^{b_{0}}$ factor in the preexponent. Hence we choose the value of $\mu_{r}$ to be set by the geometric average of the instanton sizes,

$$
\mu_{r}=1 / \sqrt{\rho \bar{\rho}},
$$

and as the result, all the running coupling constants appearing on the right-hand side of (2.24) are given by the following one-loop expression,

$$
\frac{4 \pi}{\alpha_{s}(1 / \sqrt{\rho \bar{\rho}})}=\frac{4 \pi}{\alpha_{s}(E)}-b_{0} \log \left(\rho \bar{\rho} E^{2}\right)
$$

\section{More on instanton-anti-instanton interaction}

It can be useful to separate the instanton-anti-instanton interaction potential $U_{\text {int }}$ from the total action $S_{I \bar{I}}$,

$U_{\mathrm{int}}(z)=S_{I}+S_{\bar{I}}-S_{I \bar{I}}(z)=\frac{4 \pi}{\alpha_{s}\left(\mu_{r}\right)}(1-\mathcal{S}(z))$,

where

$$
S_{I}=\frac{2 \pi}{\alpha_{s}\left(\mu_{r}\right)}=S_{\bar{I}}
$$

denote the individual actions of the single instanton and the single-anti-instanton. It then follows from our earlier 
discussion that in the limit of large separations, the interaction potential vanishes, and in the opposite limit where the individual instantons mutually annihilate, the interaction cancels the effect of the individual instanton actions,

$$
\begin{gathered}
\lim _{z \rightarrow \infty} U_{\text {int }}=\frac{6}{z^{2}}+\mathcal{O}\left(\frac{1}{z^{4}} \log z\right) \rightarrow 0, \\
\lim _{z \rightarrow 1} U_{\text {int }}=2 S_{I}\left(1-\frac{6}{5}(z-1)^{2}+\mathcal{O}\left((z-1)^{3}\right)\right) \rightarrow 2 S_{I} .
\end{gathered}
$$

The exponent of the instanton-anti-instanton action appearing in the optical theorem expression for the instanton total cross section (2.24), can be interpreted as the series expansion in powers of the instanton interaction potential,

$$
\exp \left(-\frac{4 \pi}{\alpha_{s}\left(\mu_{r}\right)} \mathcal{S}(z)\right)=\sum_{n=0}^{\infty} \frac{1}{n !}\left(U_{\mathrm{int}}\right)^{n} \exp \left(-S_{I}-S_{\bar{I}}\right),
$$

where $n$ is the number of the cut propagators in the imaginary part of the forward elastic scattering amplitude, i.e., the number of final state gluons in the instanton process. The expression (2.31) will be useful for in the following section for obtaining the mean number of final state gluons from our optical-theorem-based approach.

We should further note that the expression (2.20) given above corresponds to the action of the instanton- anti-instanton configuration for the choice of the relative orientation matrix $\Omega$ that corresponds to the maximal attraction between the instanton and the anti-instanton. In general one should integrate over all relative orientations on the right-hand side of (2.24). The result of this integration (see Appendix B) is

$$
\begin{aligned}
\int d \Omega e^{-\frac{4 \pi}{\alpha_{s}\left(\mu_{r}\right)} \mathcal{S}(z, \Omega)} & =\frac{1}{9 \sqrt{\pi}}\left(\frac{3}{U_{\mathrm{int}}}\right)^{7 / 2} e^{-\frac{4 \pi}{\alpha_{S}\left(\mu_{r}\right)} \mathcal{S}(z)} \\
& =\frac{1}{9 \sqrt{\pi}}\left(\frac{3 \alpha_{s}\left(\mu_{r}\right)}{4 \pi(1-\mathcal{S}(z))}\right)^{7 / 2} e^{-\frac{4 \pi}{\alpha_{S}\left(\mu_{r}\right)} \mathcal{S}(z)} .
\end{aligned}
$$

\section{The master integral}

We now introduce dimensionless integration variables,

$$
\begin{aligned}
& r_{0}=R_{0} E, \quad r=|\vec{R}| E, \\
& y=\rho \bar{\rho} E^{2}, \quad x=\rho / \bar{\rho},
\end{aligned}
$$

and use them to write down the instanton parton-level

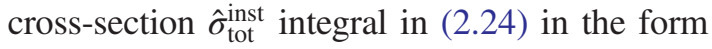

$$
\hat{\sigma}_{\text {tot }}^{\text {inst }}(E)=\frac{1}{E^{2}} \operatorname{Im} \int_{-\infty}^{+\infty} d r_{0} e^{r_{0}} G\left(r_{0}, E\right),
$$

where

$$
\begin{aligned}
G\left(r_{0}, E\right)= & \frac{\kappa^{2} \pi^{4}}{2^{17}} \sqrt{\frac{\pi}{3}} \int_{0}^{\infty} r^{2} d r \int_{0}^{\infty} \frac{d x}{x} \int_{0}^{\infty} \frac{d y}{y}\left(\frac{4 \pi}{\alpha_{s}}\right)^{21 / 2}\left(\frac{1}{1-\mathcal{S}(z)}\right)^{7 / 2} \\
& \times \mathcal{K}_{\text {ferm }}(z) \exp \left(-\frac{4 \pi}{\alpha_{s}} \mathcal{S}(z)-\frac{\alpha_{s}}{4 \pi} \frac{x+1 / x}{4} y \log y\right) .
\end{aligned}
$$

Here $\kappa, \mathcal{S}(z)$, and $\mathcal{K}_{\text {ferm }}(z)$ are given by (2.6), (2.20), (2.22), and (2.23), and the conformal ratio variable $z$ is expressed in terms of our dimensionless variables via

$z=\frac{1}{2}\left(\xi+\left(\xi^{2}-4\right)^{1 / 2}\right), \quad$ where $\xi=\frac{r_{0}^{2}+r^{2}}{y}+x+\frac{1}{x}$,

in agreement with the expression (2.19).

The final ingredient we need is the expression (2.26) for the running couplings in terms of the $y$ variable,

$$
\begin{aligned}
\frac{4 \pi}{\alpha_{s}}(y ; E) & =\frac{4 \pi}{\alpha_{s}(E)}-b_{0} \log y \\
& =\frac{4 \pi}{0.416}+2 b_{0} \log \frac{E}{1 \mathrm{GeV}}-b_{0} \log y,
\end{aligned}
$$

as follows from (2.26) and (2.34). We will thus set $\frac{4 \pi}{\alpha_{s}}=$ $\frac{4 \pi}{\alpha_{s}}(y ; E)$ in the integrand (2.36) [including the function in the exponent and the nonexponential terms in the integrant in (2.36)].

To compute the instanton cross section (2.35) we first numerically evaluate the integral (2.36) and obtain the values for $G\left(r_{0}, E\right)$ for a wide range of both arguments, $r_{0}$ and $E$. After that we perform the final integration over $r_{0}$ in (2.35) by expanding the integrand in

$$
\hat{\sigma}_{\text {tot }}^{\text {inst }}(E)=\frac{1}{E^{2}} \operatorname{Im} \int_{-\infty}^{+\infty} d r_{0} e^{r_{0}+\log G\left(r_{0}, E\right)},
$$

around the stationary point solution for $r_{0}$ of the function $r_{0}+\log G\left(r_{0}, E\right)$ in the exponent, 

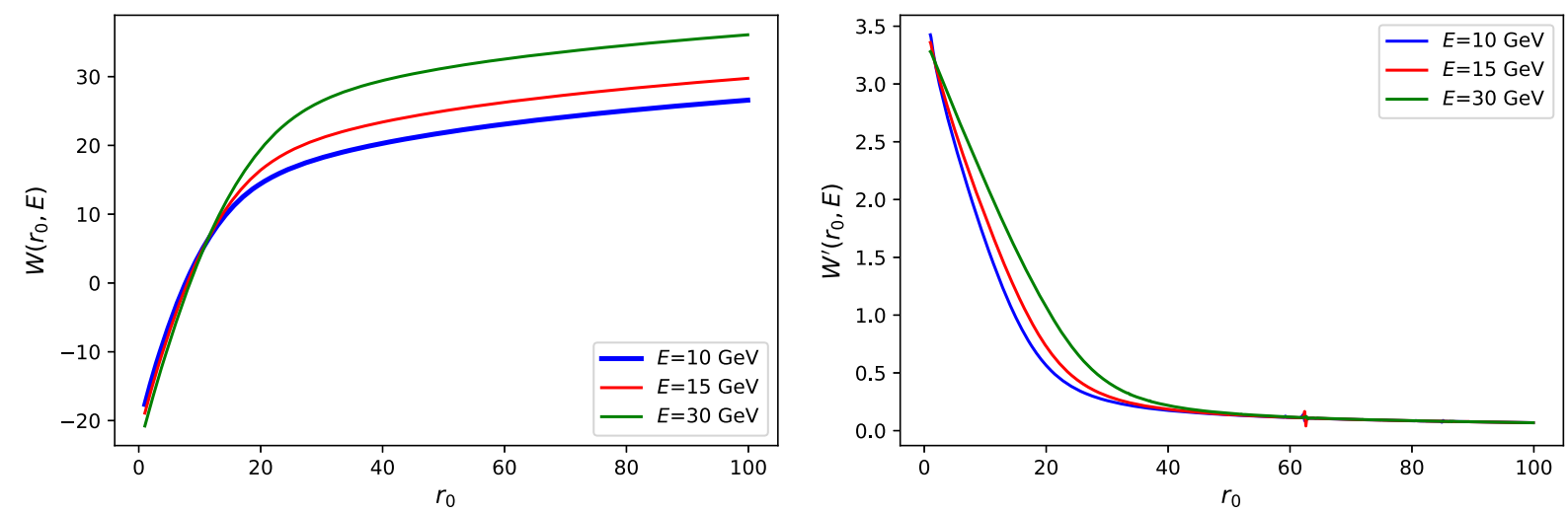

FIG. 1. Left: $W\left(r_{0}, E\right)$ plotted for $E=10,15,30 \mathrm{GeV}$ and $0<r_{0}<100$. Right: $W^{\prime}\left(r_{0}, E\right)$ plotted for $E=10$, 15, 30 GeV and $0<r_{0}<100$. NB one should ignore the small spike at $r_{0}=60$ as this is merely an artifact of the numerical accuracy of our differentiation and integration functions.

$$
r_{0}(E): \partial_{r_{0}} \log G\left(r_{0}, E\right)=-1
$$

for each value of $E$. The saddle-point evaluation of the $r_{0}$ integral (2.39) gives

$$
\begin{aligned}
\hat{\sigma}_{\mathrm{tot}}^{\text {inst }}(E) & \left.\approx \frac{1}{E^{2}} \sqrt{\frac{2 \pi}{-\partial_{r_{0}}^{2} \log G}}\right|_{r_{0}=r_{0}(E)} e^{r_{0}(E)+\log G\left(r_{0}(E), E\right)} \\
& =\left.\frac{1}{E^{2}} \sqrt{\frac{2 \pi}{W^{\prime \prime}}}\right|_{r_{0}=r_{0}(E)} e^{r_{0}(E)-W\left(r_{0}(E), E\right)},
\end{aligned}
$$

where we have defined

$$
\begin{gathered}
W\left(r_{0}, E\right)=-\log G_{0}\left(r_{0}, E\right), \\
W^{\prime}\left(r_{0}, E\right)=-\partial_{r_{0}} \log G\left(r_{0}, E\right) .
\end{gathered}
$$

The numerical integration in (2.36) was carried out using the PYTHON package SCIPY [28] for $E$ in the range in $10<$ $E<2000 \mathrm{GeV}$ and for a wide range in $r_{0}$ to accommodate a sufficiently large interval around the expected values of the saddle point $r_{0}(E)$ in (2.40). In Fig. 1 we plot the resulting functions $W\left(r_{0}, E\right)$ and $W^{\prime}\left(r_{0}, E\right)$ for fixed values of $E=10$, $15,30 \mathrm{GeV}$ in the range $0<r_{0}<100$ alongside the $r_{0}$. The function $W\left(r_{0}, E\right)$ plays the role of the effective instantonanti-instanton Euclidean action [this is because it arises from integrating the exponent of the classical action $e^{-\frac{4 \pi}{\alpha_{S}} \mathcal{S}(z)}$ over the collective coordinates of non-negative modes of the $I \bar{I}$ configuration on the RHS of (2.36)]. The saddle-point value for $r_{0}$ is given by the equation $W^{\prime}\left(r_{0}, E\right)=1$ for each fixed value of $E$, as dictated by (2.40) above.

Having determined $W\left(r_{0}, E\right)$ and its derivatives as functions of $r_{0}$ and $E$ we can now carry out the final integration over $r_{0}$ using the saddle-point approximation formula (2.41) for the imaginary part of the forward elastic scattering amplitude and hence for the partonic instanton cross section $\hat{\sigma}_{\text {tot }}^{\text {inst }}(E)$. Our final results for the partonic instanton cross section (2.35) are displayed in Table I.

Then the hadronic cross sections are calculated from these partonic cross sections using the NNPDF3.1luxQED

TABLE I. The instanton cross section presented for a range of partonic C.o.M. energies $\sqrt{\hat{s}}=E$ and the mean number of gluons at this energy calculated using Eq. (2.46).

\begin{tabular}{lccccccc}
\hline \hline$\sqrt{\hat{s}}[\mathrm{GeV}]$ & 50 & 100 & 150 & 200 & 300 & 400 & 500 \\
\hline$\left\langle n_{g}\right\rangle$ & 9.43 & 11.2 & 12.22 & 12.94 & 13.96 & 14.68 & 15.23 \\
$\hat{\sigma}_{\text {itst }}^{\text {ist }}[\mathrm{pb}]$ & $207.33 \times 10^{3}$ & $1.29 \times 10^{3}$ & 53.1 & 5.21 & $165.73 \times 10^{-3}$ & $13.65 \times 10^{-3}$ & $1.89 \times 10^{-3}$ \\
\hline \hline
\end{tabular}

TABLE II. Hadronic cross sections for QCD instanton processes at a range of colliders with different CoM energies $\sqrt{s_{p \bar{p}}}$ evaluated using Eq. (2.43). The minimal allowed partonic energy is $E_{\min }=\sqrt{\hat{s}_{\min }}$.

\begin{tabular}{lrrrrrrr}
\hline \hline$E_{\min }[\mathrm{GeV}]$ & \multicolumn{1}{c}{50} & \multicolumn{1}{c}{100} & \multicolumn{1}{c}{150} & \multicolumn{1}{c}{200} & \multicolumn{1}{c}{300} & 400 & 500 \\
\hline$\sigma_{p \bar{p} \rightarrow I} \sqrt{s_{p \bar{p}}}=1.96 \mathrm{TeV}$ & $2.62 \mu \mathrm{b}$ & $2.61 \mathrm{nb}$ & $29.6 \mathrm{pb}$ & $1.59 \mathrm{pb}$ & $6.94 \mathrm{fb}$ & $105 \mathrm{ab}$ & $3.06 \mathrm{ab}$ \\
$\sigma_{p p \rightarrow I} \sqrt{s_{p p}}=14 \mathrm{TeV}$ & $58.19 \mu \mathrm{b}$ & $129.70 \mathrm{nb}$ & $2.769 \mathrm{nb}$ & $270.61 \mathrm{pb}$ & $3.04 \mathrm{pb}$ & $114.04 \mathrm{fb}$ & $8.293 \mathrm{fb}$ \\
$\sigma_{p p \rightarrow I} \sqrt{s_{p p}}=30 \mathrm{TeV}$ & $211.0 \mu \mathrm{b}$ & $400.9 \mathrm{nb}$ & $9.51 \mathrm{nb}$ & $1.02 \mathrm{nb}$ & $13.3 \mathrm{pb}$ & $559.3 \mathrm{fb}$ & $46.3 \mathrm{fb}$ \\
$\sigma_{p p \rightarrow I} \sqrt{s_{p p}}=100 \mathrm{TeV}$ & $771.0 \mu \mathrm{b}$ & $2.12 \mu \mathrm{b}$ & $48.3 \mathrm{nb}$ & $5.65 \mathrm{nb}$ & $88.3 \mathrm{pb}$ & $4.42 \mathrm{pb}$ & $395.0 \mathrm{fb}$ \\
\hline \hline
\end{tabular}


NNLO dataset with $\alpha_{s}\left(M_{Z}\right)=0.118[29,30]$ and displayed in Table II. These are calculated using the usual formula

$$
\begin{aligned}
& \sigma_{p p \rightarrow I}\left(\hat{s}>\hat{s}_{\text {min }}\right) \\
& =\int_{\hat{s}_{\text {min }}}^{s_{p p}} d x_{1} d x_{2} f\left(x_{1}, Q^{2}\right) f\left(x_{2}, Q^{2}\right) \hat{\sigma}\left(\hat{s}=x_{1} x_{2} s_{p p}\right),
\end{aligned}
$$

where $s_{p p}$ is the center-of-mass energy of the hadron collider, $\hat{\sigma}$ is the partonic instanton cross section, and $\hat{s}_{\min }$ is the minimum invariant mass squared of the produced system. NB here we are only considering the gluon initiated process; otherwise we require a sum over such integrals.

\section{E. Mean number of final state gluons}

In our approach of computing the total partonic cross section via the optical theorem in (2.35) and (2.36) we have already effectively summed over the number gluons $n_{g}$ in the final state. This sum can be uncovered by using the series expansion (2.31) of the exponent of the instantonanti-instanton action on the right-hand side of (2.36),

$$
\begin{aligned}
G\left(r_{0}, E\right)= & \frac{\kappa^{2} \pi^{4}}{2^{17}} \sqrt{\frac{\pi}{3}} \int_{0}^{\infty} r^{2} d r \int_{0}^{\infty} \frac{d x}{x} \int_{0}^{\infty} \frac{d y}{y}\left(\frac{4 \pi}{\alpha_{s}}\right)^{21 / 2}\left(\frac{1}{1-\mathcal{S}(z)}\right)^{7 / 2} \mathcal{K}_{\text {ferm }}(z) \\
& \times \sum_{n_{g}=0}^{\infty} \frac{1}{n_{g} !}\left(U_{\text {int }}\right)^{n_{g}} \exp \left(-\frac{4 \pi}{\alpha_{s}}-\frac{\alpha_{s}}{4 \pi} \frac{x+1 / x}{4} y \log y\right) .
\end{aligned}
$$

The mean value of $n_{g}$ (i.e., the value that gives the dominant contribution to the integral) is then easily found to be given by the expectation value of the interaction potential,

$$
\left\langle n_{g}\right\rangle=\left\langle U_{\text {int }}\right\rangle,
$$

where the expectation value of $\left\langle U_{\text {int }}\right\rangle$ is obtained by inserting $U_{\text {int }}=\frac{4 \pi}{\alpha_{s}(y ; E)}(1-\mathcal{S}(z))$ into the integrand on the right-hand sides of (2.35) and (2.36) and normalizing by $1 /\left(E^{2} \hat{\sigma}_{\text {tot }}^{\text {inst }}\right)$.

In practice, we compute

$$
\begin{aligned}
\left\langle n_{g}\right\rangle= & \frac{1}{G\left(r_{0}, E\right)} \frac{\kappa^{2} \pi^{4}}{2^{17}} \sqrt{\frac{\pi}{3}} \int_{0}^{\infty} r^{2} d r \int_{0}^{\infty} \frac{d x}{x} \int_{0}^{\infty} \frac{d y}{y}\left(\frac{4 \pi}{\alpha_{s}(y ; E)}\right)^{21 / 2}\left(\frac{1}{1-\mathcal{S}(z)}\right)^{7 / 2} \\
& \times \mathcal{K}_{\text {ferm }}(z) \frac{4 \pi}{\alpha_{s}(y ; E)}(1-\mathcal{S}(z)) \cdot \exp \left(-\frac{4 \pi}{\alpha_{s}(y ; E)} \mathcal{S}(z)-\frac{\alpha_{s}(y ; E)}{4 \pi} \frac{x+1 / x}{4} y \log y\right) .
\end{aligned}
$$

On the right-hand side we have integrated over the $y, x, r$ variables. The variable $r_{0}$ is taken to be at its saddle-point value for each fixed value of the energy $E$.

To account for the possibility of the new shifted saddle point we do the following:

$$
\begin{aligned}
\left\langle n_{g}\right\rangle= & \frac{1}{\operatorname{Im} \int_{-\infty}^{+\infty} d r_{0} e^{r_{0}} G\left(r_{0}, E\right)} \\
& \times \operatorname{Im} \int_{-\infty}^{+\infty} d r_{0} e^{r_{0}} \frac{\kappa^{2} \pi^{4}}{2^{17}} \sqrt{\frac{\pi}{3}} \int_{0}^{\infty} r^{2} d r \int_{0}^{\infty} \frac{d x}{x} \int_{0}^{\infty} \frac{d y}{y}\left(\frac{4 \pi}{\alpha_{s}(y ; E)}\right)^{21 / 2}\left(\frac{1}{1-\mathcal{S}(z)}\right)^{7 / 2} \\
& \times \mathcal{K}_{\text {ferm }}(z) \frac{4 \pi}{\alpha_{s}(y ; E)}(1-\mathcal{S}(z)) \cdot \exp \left(-\frac{4 \pi}{\alpha_{s}(y ; E)} \mathcal{S}(z)-\frac{\alpha_{s}(y ; E)}{4 \pi} \frac{x+1 / x}{4} y \log y\right)
\end{aligned}
$$

\section{INSTANTON RECOIL BY A JET}

In this section we explain how to generalize the calculation of the instanton process presented above to the case where a jet is emitted from one of the initial state partons. This is of course an important process for collider studies as it allows one to recoil the instanton-generated multiparticle final state by a high- $p_{T}$ jet.

When the jet is carrying momentum $p$ produced from an initial parton $p_{1}$, the secondary gluon $q$ entering the instanton vertex will necessarily have a virtuality $q^{2}=-Q^{2} \neq 0{ }^{1}$ In the partonic center-of-mass frame we have

\footnotetext{
${ }^{1}$ In the complementary scenario where a high- $p_{T}$ jet is emitted from the instanton vertex in the final state, no virtualities arise, all momenta entering and leaving the instanton vertex are on-shell, and the formalism presented in the earlier section requires no modifications.
} 


$$
\begin{aligned}
p_{1}= & \left(\sqrt{\hat{s}} / 2,0, p_{L}\right), \quad p_{2}=\left(\sqrt{\hat{s}} / 2,0,-p_{L}\right), \\
& \text { where }\left|p_{L}\right|=\sqrt{\hat{s}} / 2, \\
p_{1}= & q+p, \quad p=\left(\left|p_{T}\right|, p_{T}, 0\right), \\
Q^{2}= & -q^{2}=-\left(p_{1}-p\right)^{2}=\sqrt{\hat{s}} p_{T} .
\end{aligned}
$$

Here we have assumed for simplicity that the jet momentum $p$ is transverse; i.e., it does not have a longitudinal component.

The kinematic-invariant CoM energy for the parton-level process is, as before, $\sqrt{\hat{s}}$, where $\hat{s}=\left(p_{1}+p_{2}\right)^{2}$. On the other hand, the invariant mass entering the instanton vertex $\sqrt{s^{\prime}}$ is now different,

$$
s^{\prime}=\left(q+p_{2}\right)^{2}=\hat{s}-2 Q^{2}=\sqrt{\hat{s}}\left(\sqrt{\hat{s}}-2 p_{T}\right) .
$$

The virtuality $Q$ of an incoming gluon leg, induced by a no-zero $p_{T}$, introduces a multiplicative form factor $e^{-Q \rho}$ into the instanton vertex. This is a well-known result $[25,27,31]$ that is a direct consequence of Fourier transforming the instanton field to the momentum space

to obtain $A_{\mathrm{LSZ}}^{\text {inst }}(q)$, where the momentum $q$ has a large virtuality, $Q^{2}$. For the instanton cross section one needs to compute, $A_{\mathrm{LSZ}}^{\mathrm{inst}}(q) A_{\mathrm{LSZ}}^{\mathrm{inst}}\left(p_{2}\right) A_{\mathrm{LSZ}}^{\overline{\mathrm{inst}}}(-q) A_{\mathrm{LSZ}}^{\overline{\mathrm{inst}}}\left(-p_{2}\right)$, in analogy with Eq. (2.18), which gives the overall form factor

$\exp (-Q(\rho+\bar{\rho}))=\exp \left(-\frac{Q}{E} \sqrt{y(x+1 / x+2)}\right)$,

that needs to be included in the integral (2.24). On the righthand side of this equation we used our standard dimensionless variables $x$ and $y$ defined in (2.33) and (2.34).

The second modification of the integral in (2.24) is that the energy variable $E$ corresponds to the instanton vertex energy $E=\sqrt{s^{\prime}}$ defined in (3.2), which is smaller than the overall invariant mass $\sqrt{\hat{s}}$ of the parton-level process.

In summary, the instanton parton-level cross section $\hat{\sigma}_{\text {tot }}^{\text {inst }}\left(\sqrt{\hat{s}}, p_{T}\right)$ is computed as follows:

(1) For each pair of physical variables $\hat{s}, p_{T}$, introduce the auxiliary variables $E$ and $Q$,

$$
Q^{2}=p_{T} \sqrt{\hat{s}}, \quad E^{2}=\hat{s}-2 Q^{2} .
$$

(2) Numerically compute the integral,

$$
\begin{aligned}
\tilde{G}\left(r_{0}, E, Q\right)= & \frac{\kappa^{2} \pi^{4}}{2^{17}} \sqrt{\frac{\pi}{3}} \int_{0}^{\infty} r^{2} d r \int_{0}^{\infty} \frac{d x}{x} \int_{0}^{\infty} \frac{d y}{y}\left(\frac{4 \pi}{\alpha_{s}}\right)^{21 / 2}\left(\frac{1}{1-\mathcal{S}(z)}\right)^{7 / 2} \\
& \times \mathcal{K}_{\text {ferm }}(z) \exp \left(-\frac{4 \pi}{\alpha_{s}} \mathcal{S}(z)-\frac{\alpha_{s}}{4 \pi} \frac{x+1 / x}{4} y \log y-\frac{Q}{E} \sqrt{y\left(x+\frac{1}{x}+2\right)}\right)
\end{aligned}
$$

and use it to evaluate the expression for the cross section,

$$
I(E, Q)=\frac{1}{E^{2}} \operatorname{Im} \int_{-\infty}^{+\infty} d r_{0} e^{r_{0}} \tilde{G}\left(r_{0}, E, Q\right),
$$

in the saddle-point approximation, as before.

(3) The cross section in physical variables is then obtained via

$$
\hat{\sigma}_{\text {tot }}^{\text {inst }}\left(\sqrt{\hat{s}}, p_{T}\right)=\left.I(E, Q)\right|_{Q^{2}=p_{T} \sqrt{\hat{s}}, E^{2}=\hat{s}-2 p_{T} \sqrt{\hat{s}}} .
$$

Table III presents the results for the instanton cross section at parton level for a range of partonic CoM energies $\sqrt{\hat{s}}$ and for a fixed value of the recoiled jet transverse momentum $p_{T}=150 \mathrm{GeV}$. The resulting cross sections fixed are negligibly small. To complement these results we have also computed instanton cross sections for the case where $p_{T}$ is scaled with the energy. Table IV presents the results at parton level where the recoiled jet transverse momentum is chosen as $p_{T}=\sqrt{\hat{s}} / 3$.

From the results in Tables IV and III we see that the cross sections calculated for the processes where the instanton recoils against a jet with large momentum are too small to be observable at any present or envisioned high-energy collider. While increasing the transverse momentum for objects that are difficult to reconstruct by

TABLE III. The instanton partonic cross section recoiled against a hard jet with $p_{T}=150 \mathrm{GeV}$ emitted from an initial state and calculated using Eq. (3.7). Results for the cross section are shown for a range of partonic CoM energies $\sqrt{\hat{s}}$.

\begin{tabular}{lcccccc}
\hline \hline$\sqrt{\hat{s}}[\mathrm{GeV}]$ & 310 & 350 & 375 & 400 & 450 & 500 \\
\hline$\hat{\sigma}_{\text {tot }}^{\text {inst }}[\mathrm{pb}]$ & $3.42 \times 10^{-23}$ & $1.35 \times 10^{-18}$ & $1.06 \times 10^{-17}$ & $1.13 \times 10^{-16}$ & $9.23 \times 10^{-16}$ & $3.10 \times 10^{-15}$ \\
\hline \hline
\end{tabular}


TABLE IV. The cross section presented for a range of partonic CoM energies $\sqrt{\hat{s}}=E$ where the recoiled $p_{T}$ is scaled with the energy, $p_{T}=\sqrt{\hat{s}} / 3$.

\begin{tabular}{lcccccc}
\hline \hline$\sqrt{\hat{s}}[\mathrm{GeV}]$ & 100 & 150 & 200 & 300 & 400 & 500 \\
\hline$\hat{\sigma}_{\mathrm{tot}}^{\text {inst }}[\mathrm{pb}]$ & $1.68 \times 10^{-7}$ & $1.20 \times 10^{-9}$ & $3.24 \times 10^{-11}$ & $1.84 \times 10^{-13}$ & $4.38 \times 10^{-15}$ & $2.38 \times 10^{-16}$ \\
\hline \hline
\end{tabular}

recoiling them against a hard object is often a popular method to improve the sensitivity of the LHC to new physics (see, e.g., [32-35]), the instanton shields itself from such an option. Consequently, the only way to obtain sensitivity to instantons is to disentangle their spherical radiation profile, made of fairly soft jets, from SM QCD backgrounds.

\section{SEARCH FOR INSTANTON EVENTS AT HADRON COLLIDERS}

\section{A. Topology of instanton events}

Since the global event topology of instanton processes is spherically symmetric, and therefore distinctly different from perturbative-QCD events, event shape observables [36] can be a powerful way to identify these processes.

The sphericity tensor is defined as

$$
S^{\alpha \beta}=\frac{\sum_{i} p_{i}^{\alpha} p_{i}^{\beta}}{\sum_{i}\left|\mathbf{p}_{i}\right|^{2}}
$$

where $\alpha, \beta$ run over spatial indices and $i$ runs over the number of particles. This tensor will have three eigenvalues $\lambda_{1} \geq \lambda_{2} \geq \lambda_{3}$, with $\lambda_{1}+\lambda_{2}+\lambda_{3}=1$. The sphericity observable is then defined as $S=\frac{3}{2}\left(\lambda_{2}+\lambda_{3}\right)$. Sphericity takes values between 0 and 1 with higher values denoting a higher degree of spherical symmetry. Therefore we would expect instanton processes to have a higher sphericity than background processes which in general have some angular dependence.

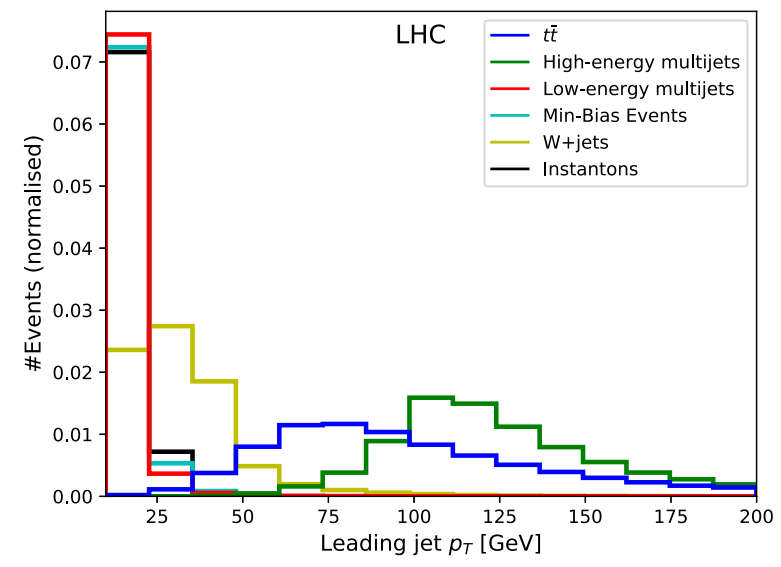

Sphericity is defined as

$$
S_{0}=\frac{\pi^{2}}{4} \min _{\vec{n}}\left(\frac{\sum_{i}\left|\vec{p}_{\perp, i} \times \vec{n}\right|}{\sum_{i}\left|\vec{p}_{\perp, i}\right|}\right)^{2}
$$

where $\vec{n}$ is a unit vector with a zero longitudinal component. Again, $S_{0}$ takes values between 0 and 1 , with 1 representing a completely isotropic event and 0 being a pencil-like event. This variable is closely related to thrust which is defined as

$$
\tau=1-\max _{\vec{n}} \frac{\sum_{i}\left|\vec{p}_{i} \cdot \vec{n}\right|}{\sum_{i}\left|\vec{p}_{i}\right|}
$$

where $\vec{n}$ is a unit vector. Thrust is 0 for pencil-like events and 0.5 for spherically symmetric events. The vector $\vec{n}$ which maximizes this expression is known as the thrust axis.

The final shape variable we consider is broadening. The thrust axis automatically divides the event into a left hemisphere, $\mathcal{L}$, and a right hemisphere, $\mathcal{R}$. Left and right broadening is then defined as

$$
\mathcal{B}_{\mathcal{L}}=\sum_{i \in \mathcal{L}} \frac{\left|\vec{p}_{i} \times \vec{n}\right|}{\sum_{i}\left|\vec{p}_{i}\right|}, \quad \mathcal{B}_{\mathcal{R}}=\sum_{i \in \mathcal{R}} \frac{\left|\vec{p}_{i} \times \vec{n}\right|}{\sum_{i}\left|\vec{p}_{i}\right|}
$$

where $\vec{n}$ is the thrust axis. Total broadening $\mathcal{B}$ is then the sum of the left and right broadening, $\mathcal{B}=\mathcal{B}_{\mathcal{L}}+\mathcal{B}_{\mathcal{R}}$, and takes values between 0 and 0.5 with 0.5 being spherically symmetric.

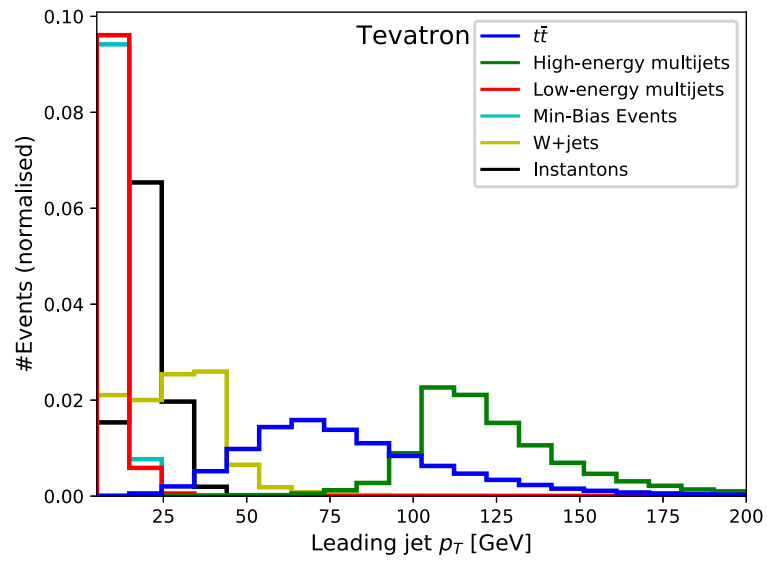

FIG. 2. The distribution of the $p_{T}$ of the leading jet for our background processes and instantons at the LHC (left) and Tevatron (right). 

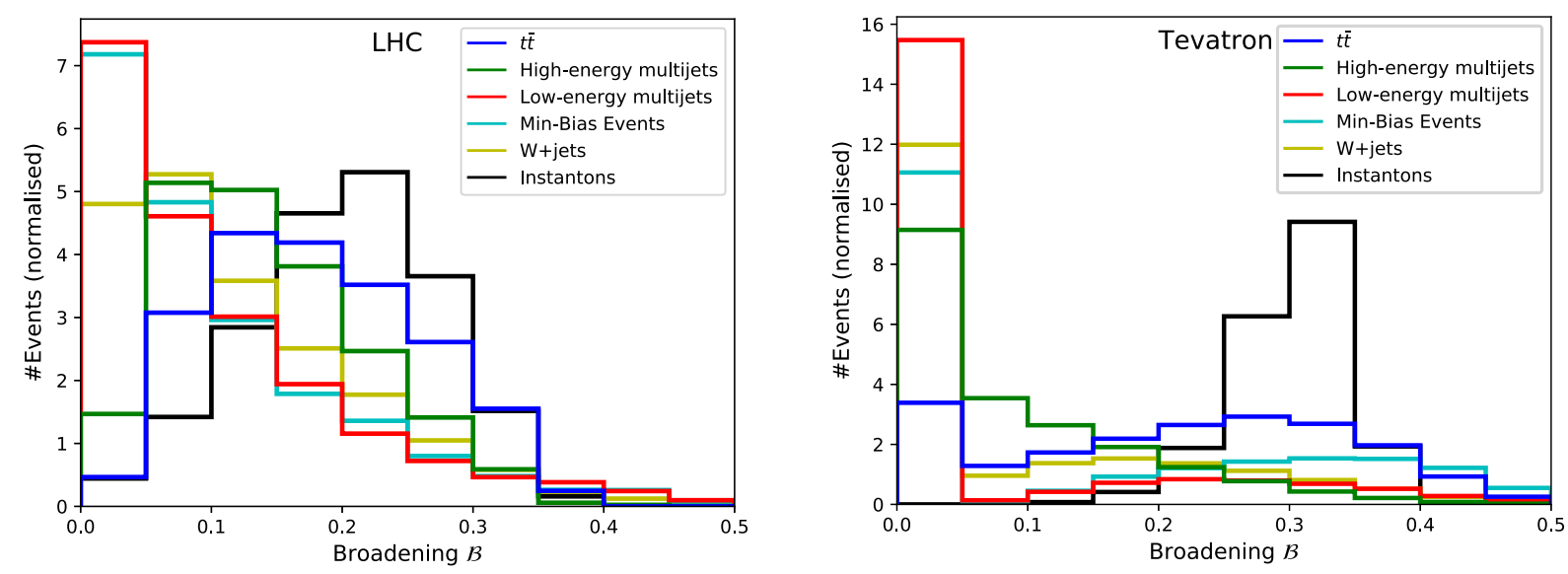

FIG. 3. The distribution of the broadening of events for our background processes and instantons at the LHC (left) and Tevatron (right).

To show the different shapes for these observables between various perturbative SM processes and instanton events at the LHC and the Tevatron, we generate the background events using PYTHIA 8 [37]. For the perturbative SM processes we consider the ones with the largest cross section and jet-rich final states, i.e., high- and low-pT multijet events, min-bias events, $t \bar{t}$ production events, and $W+$ jets events. For the signal we use RAMBO [38] to populate the phase space of the instanton final state. Each event contains four $q \bar{q}$ pairs and a Poisson-distributed number of gluons, with a mean in accordance with $n_{g}$ in Table I.

All processes are analyzed using FASTJET [39]. For the LHC we reconstruct jets using the anti- $k_{T}$ algorithm [40] with a cone size of $R=0.4$ and $p_{T} \geq 10 \mathrm{GeV}$. At the Tevatron jets were analyzed using the $k_{T}$ algorithm [40] with a cone size of $R=0.7$ and were required to have $p_{T} \geq 5 \mathrm{GeV}$. Leptons are required to have $p_{T} \geq$ $0.5 \mathrm{GeV}$. It should be noted that the instanton processes are not showered or hadronized, but this should not significantly affect the analysis as the position and energy of the reconstructed jets are conserved to a good accuracy.

We show in Fig. 2 the distribution for the $p_{T}$ of the leading jet, in Fig. 3 broadening, in Fig. 4 sphericity, and in Fig. 5 thrust for the LHC and the Tevatron, respectively. The differences in the histograms between LHC and Tevatron originate in the different jet definitions and $p_{T}$ thresholds. This leads to more spherical events and thus higher values for thrust and sphericity at the Tevatron. For the backgrounds we include the processes that have the largest perturbatively calculable cross sections. Most of these processes, in particular high-energy multijets and $W+$ jets events, show a more pronounced pencil-like structure than the instanton events. Overall, analyzing events with event shape observables provides a powerful method to discriminate instanton events from large Standard Model backgrounds.
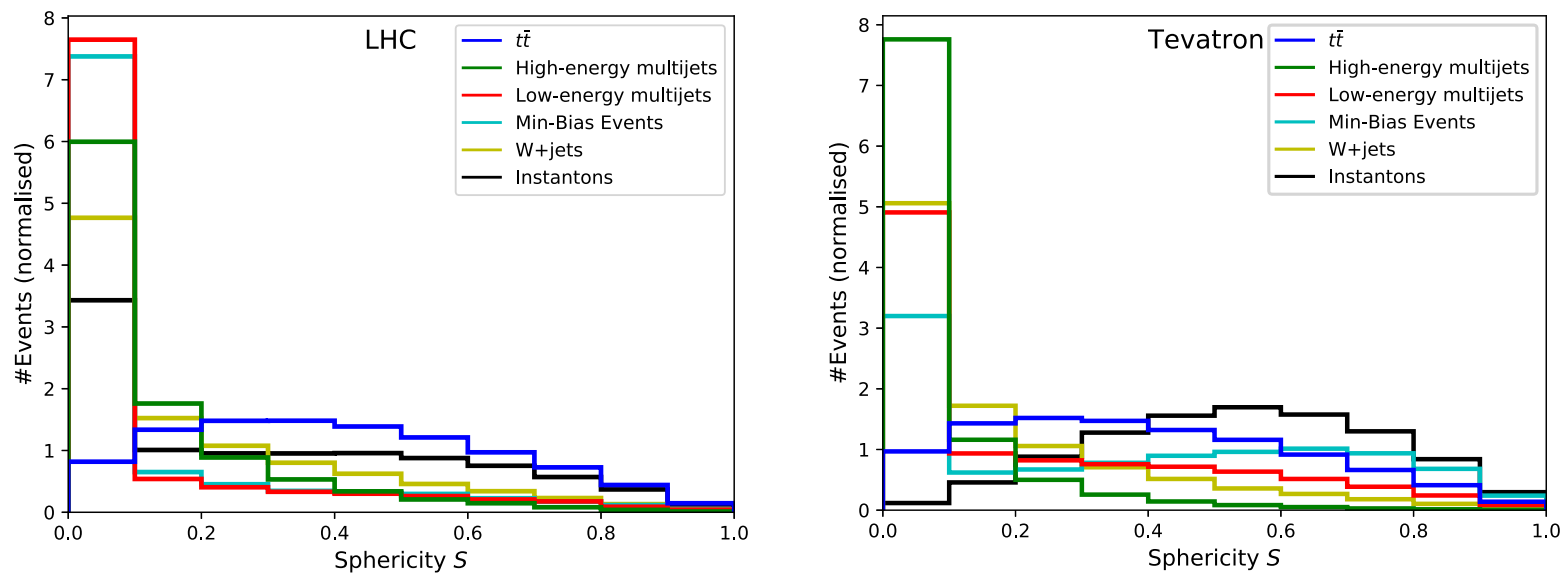

FIG. 4. The distribution of the sphericity of events for our background processes and instantons at the LHC (left) and Tevatron (right). 

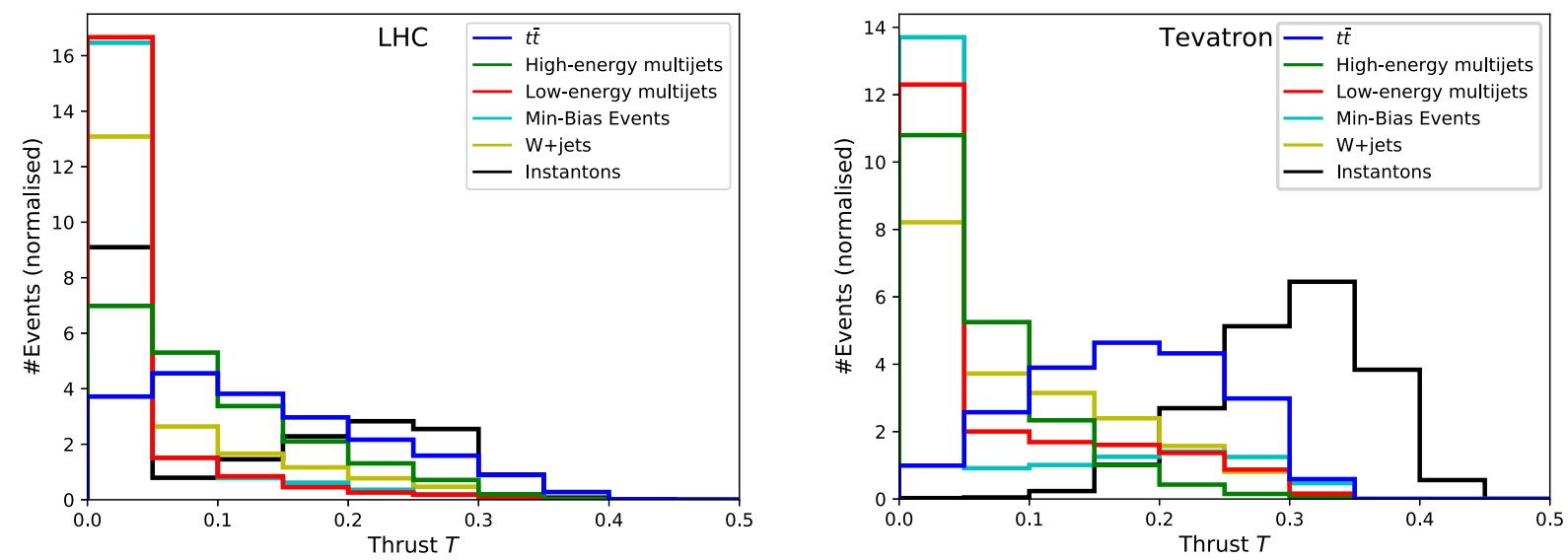

FIG. 5. The distribution of the thrust of events for our background processes and instantons at the LHC (left) and Tevatron (right).

\section{B. QCD instanton search at the LHC}

\section{Searches in high-luminosity $\mathrm{LHC}$ runs}

As a result of the trigger cuts imposed, we find that the LHC has very little sensitivity to QCD instantons in current and future high-luminosity runs. QCD instanton events produce no isolated leptons or a large amount of missing transverse energy, and so appear only as multiparticle events consisting of soft jets.

Missing transverse energy higher-level triggers require at least $E_{T \text { mis }} \geq 70 \mathrm{GeV}$ while single jet triggers are as high as $p_{T, j} \geq 360 \mathrm{GeV}$ [41]. In Sec. III we have shown that the emission of a hard jet from an initial state parton is not a viable strategy to produce an instanton. Further, the probability that one of the partons that originates in the instanton process has such a large momentum is very small as well. If one of the instanton-induced partons has a transverse momentum to pass the single-jet trigger requirements, the center-of-mass energy of the instanton $\sqrt{s^{\prime}}$ has to be at least of $\mathcal{O}(700) \mathrm{GeV}$. According to Table II, this renders the hadronic instanton cross section too small to be observable.

Thus, one would have to resort to multijet triggers, either with four jets of $p_{T, j} \geq 85 \mathrm{GeV}$ or six jets of $p_{T, j} \geq 45 \mathrm{GeV}$. Both such trigger requirements result in for instantons fairly high partonic center-of-mass energies of $\mathcal{O}(300) \mathrm{GeV}$. Generating 100000 signal events as described in Sec. IVA and reconstructing them with the anti-kT jet algorithm, we find that none of the events passes multijet triggers, which results in an upper limit on the instanton cross section that passes such trigger cuts of $\sigma_{p p \rightarrow I}^{\text {trigger }} \lesssim 10 \mathrm{fb}$. Disentangling instanton processes with less than $10 \mathrm{fb}$ of cross section from large QCD backgrounds during the event reconstruction step is a highly challenging task.

Because of increased pileup in future high-luminosity LHC runs and at future hadron colliders, e.g., the FCC-hh, trigger thresholds for jets will have to be increased, which will significantly reduce sensitivity to QCD instanton processes. Special trigger strategies would have to be developed for instantons to pass trigger requirements in such a jet-rich environment. One could speculate about the inclusion of event-shaped observables directly in the trigger strategy and a highly optimized interplay between highlevel and low-level triggers. As shown in Sec. IVA in Figs. 2-5 instanton events have a very different event topology compared to QCD-induced multijet or resonanceassociated production processes. Incorporating such observables in the trigger setup and reconstruction strategies might retain some sensitivity to instanton processes in future runs at high-energy hadron colliders.

\section{Search in low-luminosity $\mathrm{LHC}$ runs}

Rather than focusing on high-luminosity runs, we propose to pursue a different search strategy. The biggest obstacles to the discovery of QCD instanton processes are the high trigger thresholds, which are a necessity to avoid triggering on pileup in high-luminosity runs. Lowluminosity LHC runs had minimalistic trigger requirements [42], i.e., min-bias triggers which required only a single charged track with an energy of $400 \mathrm{MeV}$. Remarkably, practically all QCD instanton events would pass min-bias triggers. ATLAS and CMS [43] both are in possession of unprescaled min-bias datasets which are, however, often only used to determine the luminosity for low-pileup runs, rather than searching for new phenomena.

To assess whether these datasets can provide sensitivity to QCD instanton processes, we generate event samples as outlined in Sec. IV A with a hadronic center-of-mass energy of $\sqrt{s}=13 \mathrm{TeV}$. For the event selection we require that each event should have at least six jets $n_{j} \geq 6$ with a minimum $p_{T, j} \geq 10 \mathrm{GeV}$ and that these jets form a thrust value of $\tau \geq 0.2$. This already confidently separates instanton signal events from QCD-induced background events. For an instanton with a minimum $\sqrt{s^{\prime}} \geq 100 \mathrm{GeV}$, which can be imposed through a requirement on the invariant mass of the final state jets, we find $\frac{s}{\sqrt{b}}=50.1$ and for 
$\sqrt{s^{\prime}} \geq 200 \mathrm{GeV}$ we have $\frac{s}{\sqrt{b}}=7.1$. This shows a very good sensitivity for instanton processes in min-bias events, which can be further increased by lowering the $p_{T, j}$ requirements.

\section{QCD instanton search at the Tevatron}

We deduce from the observations in Sec. IV B 1 that future runs at high-energy high-luminosity colliders are likely to become even less sensitive to QCD instanton processes. Consequently, looking into the other direction instead, e.g., at the Tevatron, might provide yet another way to search for QCD instantons. In the top row of Table II we show the hadronic cross sections at Tevatron energies, depending on the partonic center-of-mass energy of the instanton process.

We recast several jet-rich searches and measurements by CDF [44-46]. While a large fraction of instanton events would pass the trigger criteria, the event selection criteria applied in the analysis removed the predominant fraction of instanton events. Thus, the results provided in [44-46] did not allow one to set an experimental constraint on the instanton cross section. However, if this data were reanalyzed and event reconstruction strategies following Secs. IV A and IV B 1 were applied, the Tevatron could set stringent limits on the hadronic instanton cross section.

\section{CONCLUSIONS}

Instantons are the best motivated, yet unobserved, nonperturbative effects predicted by the Standard Model. Being able to study instantons in scattering processes would provide a new window to the phenomenological exploration of the QCD vacuum, and it would allow the tensioning of nonperturbative theoretical methods developed for gauge theories with data.

In our calculation we used the optical theorem to calculate the total instanton cross section from the elastic scattering amplitude by carrying out an integral over the instanton collective coordinates, and taking into account the hard-hard initial state interactions calculated in [10]. The inclusion of these interactions is essential as it provides a cutoff for the integral over the instanton scale size $\rho$ which otherwise diverges in the IR in any QCD-like theory when no explicit external scales (such as the scalar field vacuum expectation values (VEVs), highly virtual momenta, or high temperature) are present. This theoretical approach was first presented and applied recently in [1]. We improved on the results of [1] here by using a more robust integration method by directly computing integrals over all instanton-anti-instanton collective coordinates that correspond to positive modes of the quadratic fluctuation operators in the instanton-anti-instanton background. This resulted in an increase to the instanton cross section by approximately an order of magnitude, compared to the saddle-point approximation used previously. We then also calculated the mean number of gluons in the final state using a novel and more direct approach based on computing the expectation value of the instanton-anti-instanton interaction potential.

Most of the earlier studies of QCD instanton-induced processes, prior to Ref. [1], were specific to deep-inelastic scattering (DIS) $[25,27,47]$. In this case, it was the deepinelastic momentum scale $Q$ that was essential for obtaining infrared safe instanton contributions in the DIS settings and at relatively low CoM energies. The $\mathrm{H} 1$ and ZEUS Collaborations have searched for QCD instantons at the HERA collider [48-50]. In the electroweak sector of the Standard Model, phenomenological consequences of similar nonperturbative processes were also studied in detail in the literature, including recent papers [51-53], and references therein. In particular, Ref. [54] relied on applying the theoretical formalism developed here and in [1], with the conclusion that electroweak instanton contributions at colliders are exponentially suppressed at all energies.

In this paper we have reexamined the phenomenology of QCD instanton contributions to high-energy scattering processes at hadron colliders. We showed that although the instanton cross sections are very large in a hadron collider; surprisingly such colliders have little sensitivity to instantons due to the trigger criteria necessary to reduce the data rate. Although instantons produce many final state particles, the event is isotropic and the energy is divided between all particles resulting in few particles with large $p_{T}$, one of the principle trigger requirements in a hadron collider. The higher energy instantons which could potentially pass such triggers have a vanishingly small cross section and would not be seen in sufficient numbers in the LHC to be distinguishable from the QCD background. However, examination of data collected with a minimum bias trigger $[42,43]$ showed that it should be possible to either discover instantons or severely constrain their cross section with such data, which was previously only used for luminosity calibration. We also examined data from the Tevatron and showed that certain triggers should have recorded many instanton events on tape but the selection criteria used in later analyses would render the analyses insensitive to instantons. With a new set of selection criteria this would also be another possible avenue for discovery.

\section{ACKNOWLEDGMENTS}

We thank Deepak Kar, Frank Krauss, and Matthias Schott for helpful discussions. We acknowledge funding from the STFC under Grant No. ST/P001246/1.

\section{APPENDIX A: INSTANTON-ANTI-INSTANTON VALLEY CONFIGURATION}

The forward elastic scattering amplitude is obtained from the LSZ-reduced Green's function is calculated using the path integral in the instanton-anti-instanton background, 


$$
\begin{aligned}
& G\left(p_{1}, p_{2}, p_{1}, p_{2}\right) \\
& \quad=\int D A_{\mu}[D q D \bar{q}]^{N_{f}} \prod_{i=1}^{4} A_{\mathrm{LSZ}}\left(p_{i}\right) e^{-S_{E}\left[A_{\mu}, q, \bar{q}\right]} .
\end{aligned}
$$

The definition and the meaning of the instanton-antiinstanton field configuration is provided by the valley method approach of Balitsky and Yung [8,22], and the computation of the instanton cross section using the optical theorem approach follows the approach developed in $[7,23,24,31]$ and applied to QCD instantons at proton colliders in the recent paper [1].

Usually when performing a functional integral such as this, we would expand the action around the minimum, recalling that the linear term vanishes as instantons satisfy the equations of motion, and we would get the functional determinant of $\frac{\delta^{2} S}{\delta A^{2}}$, but here we must be careful. If this operator possesses small or zero eigenvalues, then the usual $(\text { det })^{-\frac{1}{2}}$ will become very large or singular as the Gaussian approximation fails. We must treat these zero/quasizero modes carefully. These modes arise when there is a symmetry or approximate symmetry of the system leaving the action unchanged.

A typical example of a zero mode is the center of the BPST instanton, the corresponding collective coordinate $x_{0}$ does not affect the value of the instanton action, and so translation is a symmetry. In general each symmetry of the system that is broken by the background field configuration (in our case the instanton) will have an associated collective coordinate, $\tau$, with zero mode $\frac{\partial A^{\mathrm{cl}(\tau)}}{\partial \tau}$, where $A^{\mathrm{cl}(\tau)}$ denotes the background field.

Quasizero modes can be understood in a similar fashion even though they do not correspond to an exact symmetry of the system. A typical example of a quasizero mode is the separation between the positions of the instanton and the anti-instanton in the instantonanti-instanton configuration. At large separations, the individual (anti-)instantons interact very weakly and the collective coordinate that corresponds to their separation becomes a nearly flat direction of the instanton-antiinstanton action. Once again we denote the background instanton-anti-instanton field configuration $A^{\mathrm{cl}(\tau)}$ and the quasizero mode is given by $\frac{\partial A^{\mathrm{cl}(\tau)}}{\partial \tau}$. In general $\tau$ will now denote the set of all collective coordinates, for the zero and quasizero modes.

The background field configuration with a quasizero mode (i.e., a nearly flat direction in the action parametrized by the $\tau$ coordinate) can now be defined as a solution of the gradient flow equation, also known as the valley equation of Balitsky and Yung [8,22],

$$
\left.\frac{\delta S}{\delta A}\right|_{A=A^{\mathrm{c}(\tau)}} \propto \epsilon^{2}(\tau) \frac{\partial A^{\mathrm{cl}(\tau)}}{\partial \tau} .
$$

If the background field is an exact classical solution, then the $\tau$-collective-coordinate parametrizes an exact zero mode and we have $\epsilon^{2}(\tau)=0$ so the valley equation collapses to the Euler-Lagrange equation. However, in the case of a quasizero mode, $\tau$ is a pseudoflat direction; the action is not at the exact minimum at any fixed value of $\tau$. In this case Eq. (A5) holds with a nonvanishing but small right-hand side, so that $\epsilon^{2}(\tau) \ll 1$. The smallness of the parameter $\epsilon^{2}(\tau)$ characterizes how flat the corresponding quasizero mode is.

To proceed with our calculation of Green's function one uses the Fadeev-Popov procedure [8,22]:

$$
\begin{aligned}
1= & \int d \tau\left|\operatorname{det}\left(\frac{d}{d \tau}\left\langle A-A^{\mathrm{cl}(\tau)}, \frac{\partial A^{\mathrm{cl}(\tau)}}{\partial \tau}\right\rangle_{w}\right)\right| \\
& \times \delta\left(\left\langle A-A^{\operatorname{cl}(\tau)}, \frac{\partial A^{\mathrm{cl}(\tau)}}{\partial \tau}\right\rangle_{w}\right) \\
= & \int d \tau \operatorname{det}\left(\left\langle\frac{\partial A^{\mathrm{cl}(\tau)}}{\partial \tau}, \frac{\partial A^{\mathrm{cl}(\tau)}}{\partial \tau}\right\rangle_{w}\right) \\
& \times \delta\left(\left\langle A-A^{\operatorname{cl}(\tau)}, \frac{\partial A^{\mathrm{cl}(\tau)}}{\partial \tau}\right\rangle_{w}\right),
\end{aligned}
$$

where $A^{\mathrm{cl}(\tau)}$ is the minimum of the action for fixed $\tau$ and $\langle A, B\rangle_{w}$ denotes the scalar product or an overlap of two field configurations,

$$
\langle A, B\rangle_{w}=\int d^{4} x w(x) A(x) B(x) .
$$

Note that the definition of the overlap above uses a positive weight function $w(x)$-the freedom to choose a convenient form of $w(x)$ is a well-known simplifying feature used in path integral expansions around instantons $[5,8,55]$ and will be utilized in what follows. Taking into account the weight factor, the valley equation reads

$$
\left.\frac{\delta S}{\delta A}\right|_{A=A^{\mathrm{cl}(\tau)}}=\epsilon^{2}(\tau) w(x) \frac{\partial A^{\mathrm{cl}(\tau)}}{\partial \tau}
$$

Inserting one of the factors of 1 in the form (A3) for each collective coordinate, and expanding the action $S(A)$ around the background field $A^{\mathrm{cl}(\tau)}$,

$$
\begin{aligned}
S(A)= & S\left(A^{\mathrm{cl}(\tau)}\right)+\left\langle\frac{\delta S\left(A^{\mathrm{cl}(\tau)}\right)}{\delta A},\left(A-A^{\mathrm{cl}(\tau)}\right)\right\rangle_{w} \\
& +\frac{1}{2}\left\langle\left(A-A^{\mathrm{cl}(\tau)}\right), \square\left(A^{\mathrm{cl}(\tau)}\right)\left(A-A^{\mathrm{cl}(\tau)}\right)\right\rangle_{w} \\
& +\cdots,
\end{aligned}
$$

we get 


$$
\begin{aligned}
G= & N \int \prod_{i} d \tau_{i} \operatorname{det}\left(\left\langle\frac{\partial A_{\tau_{i}}}{\partial \tau}, \frac{\partial A_{\tau_{j}}}{\partial \tau}\right\rangle_{w}\right) \\
& \times \int D A \prod_{i} \delta\left(\left\langle A-A^{\mathrm{cl}(\tau)}, \frac{\partial A^{\mathrm{cl}(\tau)}}{\partial \tau_{i}}\right\rangle_{w}\right) \\
& \times \prod_{m=1}^{4} A_{\mathrm{LSZ}}\left(p_{m}\right) e^{-S\left(A^{\mathrm{cl}(\tau)}\right)-\frac{1}{2}\left\langle\left(A-A^{\mathrm{cl}(\tau)}\right), \square\left(A^{\mathrm{cl}(\tau)}\right)\left(A-A^{\mathrm{cl}(\tau)}\right)\right\rangle_{w}}
\end{aligned}
$$

where $\square\left(A^{\mathrm{cl}(\tau)}\right)=\left.\frac{\delta^{2} S}{\delta A^{2}}\right|_{A=A^{\mathrm{cl}(\tau)}}$. We note that the term linear in fluctuations in the expansion of the action [the second term on the right-hand side of (A6)] in fact does not contribute to the integral in (A7). Indeed, the valley equation (A5) requires that $\delta S / \delta A$ is proportional to $\partial A / \partial \tau$ when computed on our background configuration $A^{\mathrm{cl}(\tau)}$ and then the delta function in the integrand (A7) ensures that this linear term vanishes.

Now we can perform the functional integration [8],

$$
\begin{aligned}
G= & N \int \prod_{i} d \tau_{i} \operatorname{det}\left(\left\langle\frac{\partial A_{\tau_{i}}}{\partial \tau}, \frac{\partial A_{\tau_{j}}}{\partial \tau}\right\rangle_{w}\right) \\
& \times \operatorname{det}^{-1 / 2}\left(\left\langle\frac{\partial A_{\tau}}{\partial \tau_{i}}, \square^{-1}\left(A_{\tau}\right) \frac{\partial A_{\tau}}{\partial \tau_{j}}\right\rangle\right) \\
& \times \operatorname{det}^{-1 / 2}\left(\square\left(A_{\tau}\right)\right) \prod_{m=1}^{4} A_{\mathrm{LSZ}}\left(p_{m}\right) e^{-S\left(A^{\mathrm{cl}(\tau)}\right)} .
\end{aligned}
$$

Since the $\frac{\partial A^{\mathrm{cl}(\tau)}}{\partial \tau}$ play the role of zero and quasizero modes of the action, they are the eigenfunctions of $\square\left(A^{\mathrm{cl}(\tau)}\right)$, and so

$$
\square\left(A^{\mathrm{cl}(\tau)}\right) \frac{\partial A^{\mathrm{cl}(\tau)}}{\partial \tau_{i}}=\lambda_{i} \frac{\partial A^{\mathrm{cl}(\tau)}}{\partial \tau_{i}} .
$$

This equation is valid at the leading order in the small parameter $\epsilon^{2}$ and follows from differentiating both sides of the valley equation with respect to $\tau$ and neglecting the $\epsilon^{2}(\tau) \partial^{2} A^{\mathrm{cl}(\tau)} / \partial \tau^{2}$ term.

This allows us to simplify the product of the three determinants in (A8) into

$\operatorname{det}^{1 / 2}\left(\left\langle\frac{\partial A^{\mathrm{cl}(\tau)}}{\partial \tau_{i}}, \frac{\partial A^{\mathrm{cl}(\tau)}}{\partial \tau_{j}}\right\rangle\right)\left(\operatorname{det}^{(2 p)}\left(\square\left(A^{\mathrm{cl}(\tau)}\right)\right)\right)^{-1 / 2}$,

where $\operatorname{det}^{(2 p)}$ denotes the determinant with the $2 p$ zero and quasizero modes $\left\{\lambda_{i}\right\}_{i=1}^{2 p}$ removed ( $p$ modes for the instantons and $p$ modes for the anti-instanton).

To the leading order in the small- $\epsilon$ expansion we can also factorize the quadratic fluctuation determinant in the instanton-anti-instanton background $A^{\mathrm{cl}(\tau)}=A_{\bar{I}}$ into the product of the instanton and the anti-instanton quadratic fluctuation determinants, $\operatorname{det}^{(2 p)}\left(\square\left(A_{\bar{I}}\right)\right) \approx$ $\operatorname{det}^{(p)}\left(\square\left(A_{I}\right)\right) \operatorname{det}^{(p)}\left(\square\left(A_{\bar{I}}\right)\right)$.
This gives us finally [8]

$$
G=\int d \mu_{1} d \mu_{2} \prod_{m=1}^{4} A_{\mathrm{LSZ}}\left(p_{m}\right) e^{-S\left(A^{\mathrm{cl}(\tau)}\right)},
$$

where

$$
\begin{aligned}
d \mu_{a}= & N \prod_{i=1}^{p} d \tau_{a, i} \operatorname{det}^{1 / 2}\left(\left\langle\frac{\partial A_{a}}{\partial \tau_{a, i}}, \frac{\partial A_{a}}{\partial \tau_{a, j}}\right\rangle\right) \\
& \times\left(\operatorname{det}^{(p)}\left(\square\left(A_{a}\right)\right)\right)^{-1 / 2}
\end{aligned}
$$

are the instanton and anti-instanton collective coordinate integration measures.

Having established the form of the collective coordinate integrals for the instanton-anti-instanton case, what is left for us to determine is the instanton-anti-instanton configuration itself and in particular its action as the function of (anti-)instanton collective coordinates.

The instanton-anti-instanton valley trajectory $A_{\mu}^{I \bar{I}}$ was obtained in Ref. [8] by finding an exact solution of the valley equation (A5) for a particular choice of the weight function $w(x)$ by exploring conformal invariance of the classical Yang-Mills action. The action on this configuration was computed in [23] and $[7,24]$, and it takes the form

$$
\begin{aligned}
S_{\bar{I}}(z)= & \frac{16 \pi^{2}}{g^{2}}\left(3 \frac{6 z^{2}-14}{(z-1 / z)^{2}}-17\right. \\
& \left.-3 \log (z)\left(\frac{(z-5 / z)(z+1 / z)^{2}}{(z-1 / z)^{3}}-1\right)\right),
\end{aligned}
$$

where the variable $z$ is the conformal ratio of the (anti-)instanton collective coordinates,

$z=\frac{R^{2}+\rho^{2}+\bar{\rho}^{2}+\sqrt{\left(R^{2}+\rho^{2}+\bar{\rho}^{2}\right)^{2}-4 \rho^{2} \bar{\rho}^{2}}}{2 \rho \bar{\rho}}$.

$z$ plays the role of the single negative quasizero mode of the instanton-anti-instanton valley configuration.

In the limit of the large separation between the instanton centers, $R / \rho, R / \bar{\rho} \rightarrow \infty$, the conformal ratio $z \rightarrow$ $R^{2} / \rho \bar{\rho} \rightarrow \infty$, and instanton-anti-instanton action $S_{\bar{I}}(z)$ becomes the sum of the individual instanton and antiinstanton actions,

$$
\lim _{z \rightarrow \infty} S_{I \bar{I}}(z)=\frac{8 \pi^{2}}{g^{2}}+\frac{8 \pi^{2}}{g^{2}}+\mathcal{O}\left(1 / z^{2}\right)=\frac{16 \pi^{2}}{g^{2}}
$$

and

$$
A_{\mu}^{I \bar{I}}(x) \rightarrow A_{\mu}^{I}\left(x-x_{0}\right)+A_{\mu}^{\bar{I}}\left(x-x_{0}-R\right) .
$$

In the opposite limit of a vanishing separation between the instanton centers, $R / \rho, R / \bar{\rho} \rightarrow 0$, the conformal ratio $z \rightarrow 1$ and the expression for the action $\mathcal{S}(z)$ goes to zero. This is in agreement with the expectation that in this limit the 


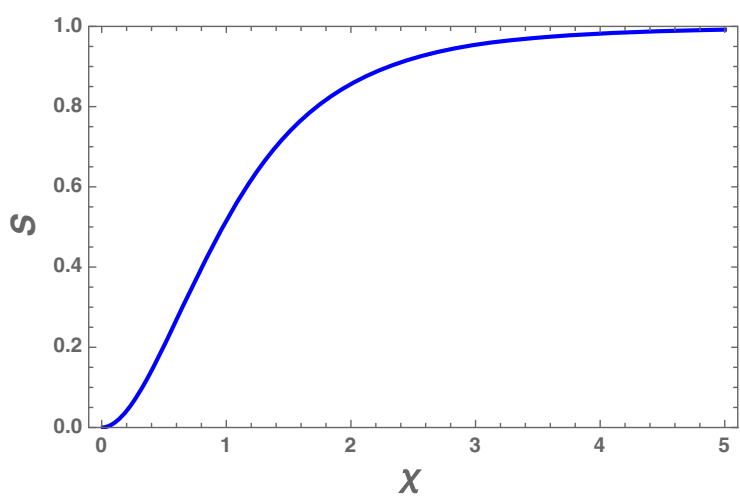

FIG. 6. The action (2.20) of the instanton-anti-instanton configuration as the function of $\chi=R / \rho$ in units of $16 \pi^{2} / g^{2}$. $S_{I \bar{I}}$ approaches the sum of the individual instanton actions at $\chi \rightarrow$ $\infty$ where the instanton interaction vanishes, and $S_{\bar{I}} \rightarrow 0$ at $\chi \rightarrow 0$ where the instanton and the anti-instanton mutually annihilate.

instanton and the anti-instanton annihilate to the perturbative vacuum $A_{\mu}=0$.

We can plot the action $S_{\bar{I}}$ as the function of the separation between the instanton centers $R$ normalized by the instanton scale sizes. For simplicity, if we assume that the sizes are equal, $\rho=\bar{\rho}$, we can write down the action $S_{I \bar{I}}$ as the function of the variable $\chi=R / \rho$. It is plotted in Fig. 6 in units of $16 \pi^{2} / g^{2}$.

\section{APPENDIX B: INTEGRATION OVER THE RELATIVE ORIENTATIONS}

To be able to integrate over relative orientations in the internal $S U(3)$ space, we need to know the form of the instanton-anti-instanton action for arbitrary values of their relative orientation matrix $\Omega$. However, our exact valley configuration is only known for the maximally attractive channel, i.e., where the interaction potential $U_{\text {int }}(z, \Omega)$ is maximized over the relative orientations at each fixed value of $z$.

What is known, however, is the form of the interaction potential $U_{\text {int }}(z, \Omega)$ in the limit of large separations. In this large-separations regime (i.e., $z \gg 1$ ) the instanton and the anti-instanton are known to have dipole-dipole interactions [56],

$$
U_{\mathrm{int}}(z, \Omega)=\frac{1}{z^{2}}\left(2 \operatorname{tr} O \operatorname{tr} O^{\dagger}-\operatorname{tr}\left(O O^{\dagger}\right)\right)+\mathcal{O}\left(\frac{1}{z^{4}} \log z\right),
$$

where $O$ is the $2 \times 2$ matrix in the upper-left corner of the $3 \times 3$ matrix $\Omega$ describing the relative instanton-antiinstanton orientation. ${ }^{2}$

Lacking the precise solution of the instanton-antiinstanton valley for general orientations at arbitrary separations, we will simply assume that the full interaction potential can always be written in the form [cf. (2.29)]

$$
U_{\mathrm{int}}(z, \Omega)=U_{\mathrm{int}}(z) \frac{1}{6}\left(2 \operatorname{tr} O \operatorname{tr} O^{\dagger}-\operatorname{tr}\left(O O^{\dagger}\right)\right),
$$

where $U_{\text {int }}(z)$ is the maximally attractive-orientation potential (2.27), (2.20). Clearly at large separations, to order $1 / z^{2}$ this expression coincides with the known dipole-dipole interaction.

We can now represent the integral over the relative orientations as follows:

$$
\int d \Omega e^{-\frac{4 \pi}{\alpha_{S}\left(\mu_{r}\right)} \mathcal{S}(z, \Omega)}=e^{-\frac{4 \pi}{\alpha_{S}\left(\mu_{r}\right)}} \int d \Omega e^{U_{\mathrm{int}}(z) \frac{1}{6}\left(2 \operatorname{tr} O \operatorname{tr} O^{\dagger}-\operatorname{tr}\left(O O^{\dagger}\right)\right) .}
$$

These types of integrals over $S U(3)$ matrices have been previously computed in the instanton literature [see Eq. (2.15) in [25] ]:

$$
\int d \Omega e^{\lambda\left(2 \operatorname{tr} O \operatorname{tr} O^{\dagger}-\operatorname{tr}\left(O O^{\dagger}\right)\right)}=\frac{1}{9 \sqrt{\pi}}(2 \lambda)^{-7 / 2} e^{6 \lambda} .
$$

Substituting $\lambda=\frac{1}{6} U_{\text {int }}(z)$ to the expression above, we now obtain the answer for our relative orientation integral in (B3),

$$
\int d \Omega e^{-\frac{4 \pi}{\alpha_{s}\left(u_{r}\right)} \mathcal{S}(z, \Omega)}=\frac{1}{9 \sqrt{\pi}}\left(\frac{3}{U_{\mathrm{int}}(z)}\right)^{7 / 2} e^{-\frac{4 \pi}{\alpha_{s}\left(u_{r}\right)} \mathcal{S}(z)}
$$

which agrees with the expression (2.32) quoted in Sec. II.

\footnotetext{
${ }^{2}$ The upper-left corner is selected by placing the instanton in the upper-left corner while allowing the anti-instanton to be anywhere in the $S U(3)$ internal space.
}

[1] V. V. Khoze, F. Krauss, and M. Schott, Large effects from small QCD instantons: Making soft bombs at hadron colliders, J. High Energy Phys. 04 (2020) 201.

[2] C. G. Callan, Jr., R. F. Dashen, and D. J. Gross, The structure of the gauge theory vacuum, Phys. Lett. 63B, 334 (1976).
[3] R. Jackiw and C. Rebbi, Vacuum Periodicity in a YangMills Quantum Theory, Phys. Rev. Lett. 37, 172 (1976).

[4] A. A. Belavin, A. M. Polyakov, A. S. Schwartz, and Yu. S. Tyupkin, Pseudoparticle solutions of the Yang-Mills equations, Phys. Lett. 59B, 85 (1975). 
[5] G. 't Hooft, Computation of the quantum effects due to a four-dimensional pseudoparticle, Phys. Rev. D 14, 3432 (1976); 18, 2199(E) (1978).

[6] G. 't Hooft, Symmetry Breaking Through Bell-Jackiw Anomalies, Phys. Rev. Lett. 37, 8 (1976).

[7] V. V. Khoze and A. Ringwald, Nonperturbative contribution to total cross-sections in non-Abelian gauge theories, Phys. Lett. B 259, 106 (1991).

[8] A. V. Yung, Instanton vacuum in supersymmetric QCD, Nucl. Phys. B297, 47 (1988).

[9] A.H. Mueller, Leading power corrections to the semiclassical approximation for gauge meson collisions in the one instanton sector, Nucl. Phys. B353, 44 (1991).

[10] A. H. Mueller, First quantum corrections to gluon-gluon collisions in the one instanton sector, Nucl. Phys. B348, 310 (1991).

[11] S. Knapen, S. Pagan Griso, M. Papucci, and D. J. Robinson, Triggering soft bombs at the LHC, J. High Energy Phys. 08 (2017) 076.

[12] S. Coleman, Aspects of Symmetry: Uses of Instantons (Cambridge University Press, Cambridge, England, 1985).

[13] A. I. Vainshtein, V. I. Zakharov, V. A. Novikov, and M. A. Shifman, ABC's of instantons, Usp. Fiz. Nauk 136, 553 (1982) [Sov. Phys. Usp. 25, 195 (1982)].

[14] M. P. Mattis, The riddle of high-energy baryon number violation, Phys. Rep. 214, 159 (1992).

[15] T. Schafer and E. V. Shuryak, Instantons in QCD, Rev. Mod. Phys. 70, 323 (1998).

[16] N. Dorey, T. J. Hollowood, V. V. Khoze, and M. P. Mattis, The calculus of many instantons, Phys. Rep. 371, 231 (2002).

[17] G. 't Hooft, How instantons solve the U(1) problem, Phys. Rep. 142, 357 (1986).

[18] A. Hasenfratz and P. Hasenfratz, The scales of Euclidean and Hamiltonian lattice QCD, Nucl. Phys. B193, 210 (1981).

[19] M. Luscher, A semiclassical formula for the topological susceptibility in a finite space-time volume, Nucl. Phys. B205, 483 (1982).

[20] A. Ringwald, High-energy breakdown of perturbation theory in the electroweak instanton sector, Nucl. Phys. B330, 1 (1990).

[21] P. B. Arnold and L. D. McLerran, Sphalerons, small fluctuations and baryon number violation in electroweak theory, Phys. Rev. D 36, 581 (1987).

[22] I. I. Balitsky and A. V. Yung, Collective-Coordinate method for quasizero modes, Phys. Lett. 168B, 113 (1986).

[23] V. V. Khoze and A. Ringwald, Valley trajectories in gauge theories, CERN Report No. CERN-TH-6082-91, 1991, https://lib-extopc.kek.jp/preprints/PDF/1991/9106/9106100 .pdf.

[24] J. Verbaarschot, Streamlines and conformal invariance in Yang-Mills theories, Nucl. Phys. B362, 33 (1991); Erratum, Nucl. Phys. B386, 236 (1992).

[25] I. I. Balitsky and V. M. Braun, Instanton-induced contributions of fractional twist in the cross-section of hard gluongluon scattering in QCD, Phys. Rev. D 47, 1879 (1993).

[26] E. V. Shuryak and J. Verbaarschot, On Baryon Number Violation and Nonperturbative Weak Processes at SSC Energies, Phys. Rev. Lett. 68, 2576 (1992).
[27] A. Ringwald and F. Schrempp, Instanton induced crosssections in deep inelastic scattering, Phys. Lett. B 438, 217 (1998).

[28] P. Virtanen, R. Gommers, T. E. Oliphant, M. Haberland, T. Reddy, D. Cournapeau et al., SciPy 1.0: Fundamental algorithms for scientific computing in Python, Nat. Methods 17, 261 (2020).

[29] A. Buckley, J. Ferrando, S. Lloyd, K. Nordstrom, B. Page, M. Refenacht, M. Schonherr, and G. Watt, LHAPDF6: Parton density access in the LHC precision era, Eur. Phys. J. C 75, 132 (2015).

[30] V. Bertone, S. Carrazza, N. P. Hartland, and J. Rojo (NNPDF Collaboration), Illuminating the photon content of the proton within a global PDF analysis, SciPost Phys. 5, 008 (2018).

[31] V. V. Khoze and A. Ringwald, Total cross-section for anomalous fermion number violation via dispersion relation, Nucl. Phys. B355, 351 (1991).

[32] G. D. Kribs, A. Martin, T. S. Roy, and M. Spannowsky, Discovering the Higgs boson in new physics events using jet substructure, Phys. Rev. D 81, 111501 (2010).

[33] M. Schlaffer, M. Spannowsky, M. Takeuchi, A. Weiler, and C. Wymant, Boosted Higgs shapes, Eur. Phys. J. C 74, 3120 (2014).

[34] P. Harris, V. V. Khoze, M. Spannowsky, and C. Williams, Constraining dark sectors at colliders: Beyond the effective theory approach, Phys. Rev. D 91, 055009 (2015).

[35] P. Harris, V. V. Khoze, M. Spannowsky, and C. Williams, Closing up on dark sectors at colliders: From 14 to $100 \mathrm{TeV}$, Phys. Rev. D 93, 054030 (2016).

[36] A. Banfi, G. P. Salam, and G. Zanderighi, Resummed event shapes at hadron-hadron colliders, J. High Energy Phys. 08 (2004) 062.

[37] T. Sjöstrand, S. Ask, J. R. Christiansen, R. Corke, N. Desai, P. Ilten, S. Mrenna, S. Prestel, C. O. Rasmussen, and P.Z. Skands, An introduction to PYTHIA 8.2, Comput. Phys. Commun. 191, 159 (2015).

[38] R. Kleiss, W. J. Stirling, and S. D. Ellis, A new Monte Carlo treatment of multiparticle phase space at high-energies, Comput. Phys. Commun. 40, 359 (1986).

[39] M. Cacciari, G. P. Salam, and G. Soyez, FastJet user manual, Eur. Phys. J. C 72, 1896 (2012).

[40] M. Cacciari, G. P. Salam, and G. Soyez, The anti- $k_{t}$ jet clustering algorithm, J. High Energy Phys. 04 (2008) 063.

[41] M. Aaboud et al. (ATLAS Collaboration), Performance of the ATLAS trigger system in 2015, Eur. Phys. J. C 77, 317 (2017).

[42] ATLAS Collaboration, Luminosity determination for lowpileup datasets at $\sqrt{s}=5$ and $13 \mathrm{TeV}$ using the ATLAS detector at the LHC.

[43] CMS Collaboration, CMS luminosity measurement for the 2017 data-taking period at $\sqrt{s}=13 \mathrm{TeV}$.

[44] T. Aaltonen et al. (CDF Collaboration), Search for resonant production of $t \bar{t}$ decaying to jets in $p \bar{p}$ collisions at $\sqrt{s}=1.96 \mathrm{TeV}$, Phys. Rev. D 84, 072003 (2011).

[45] T. Aaltonen et al. (CDF Collaboration), Top-Quark Mass Measurement Using Events with Missing Transverse Energy and Jets at CDF, Phys. Rev. Lett. 107, 232002 (2011). 
[46] T. Aaltonen et al. (CDF Collaboration), Measurements of the Top-Quark Mass and the $t \bar{t}$ Cross Section in the Hadronic $\tau+$ Jets Decay Channel at $\sqrt{s}=1.96 \mathrm{TeV}$, Phys. Rev. Lett. 109, 192001 (2012).

[47] A. Ringwald, From QCD instantons at HERA to electroweak B + L violation at VLHC, Proc. Sci., jhw2002 (2002) 008 [arXiv:hep-ph/0302112].

[48] C. Adloff et al. (H1 Collaboration), Search for QCD instanton induced processes in deep inelastic scattering at HERA, Eur. Phys. J. C 25, 495 (2002).

[49] S. Chekanov et al. (ZEUS Collaboration), Search for QCD instanton induced events in deep inelastic ep scattering at HERA, Eur. Phys. J. C 34, 255 (2004).

[50] V. Andreev et al. (H1 Collaboration), Search for QCD instanton-induced processes at HERA in the high- $Q^{2}$ domain, Eur. Phys. J. C 76, 381 (2016).
[51] A. Ringwald, K. Sakurai, and B. R. Webber, Limits on electroweak instanton-induced processes with multiple boson production, J. High Energy Phys. 11 (2018) 105.

[52] A. Papaefstathiou, S. Platzer, and K. Sakurai, On the phenomenology of sphaleron-induced processes at the LHC and beyond, J. High Energy Phys. 12 (2019) 017.

[53] J. Ellis, K. Sakurai, and M. Spannowsky, Search for sphalerons: IceCube vs LHC, J. High Energy Phys. 05 (2016) 085.

[54] V. V. Khoze and D. L. Milne, Suppression of electroweak instanton processes in high-energy collisions, arXiv: 2011.07167.

[55] H. Levine and L. G. Yaffe, Higher order instanton effects, Phys. Rev. D 19, 1225 (1979).

[56] C. G. Callan, Jr., R. F. Dashen, and D. J. Gross, Toward a theory of the strong interactions, Phys. Rev. D 17, 2717 (1978). 